\title{
The Mira variable S Orionis: relationships between the photosphere, molecular layer, dust shell, and SiO maser shell at 4 epochs $\star, \star \star, \star \star \star, \dagger$
}

\author{
M. Wittkowski ${ }^{1}$, D. A. Boboltz ${ }^{2}$, K. Ohnaka ${ }^{3}$, T. Driebe ${ }^{3}$, and M. Scholz ${ }^{4,5}$ \\ 1 European Southern Observatory, Karl-Schwarzschild-Str. 2, 85748 Garching bei München, Germany \\ e-mail: mwittkow@eso.org \\ 2 United States Naval Observatory, 3450 Massachusetts Avenue, NW, Washington, DC 20392-5420, USA \\ e-mail: dboboltz@usno.navy.mil \\ 3 Max-Planck-Institut für Radioastronomie, Auf dem Hügel 69, 53121 Bonn, Germany \\ ${ }^{4}$ Institut für Theoretische Astrophysik der Univ. Heidelberg, Albert-Ueberle-Str. 2, 69120 Heidelberg, Germany \\ 5 Institute of Astronomy, School of Physics, University of Sydney, Sydney NSW 2006, Australia \\ Received 25 January 2007 / Accepted 18 April 2007
}

\begin{abstract}
Aims. We present the first multi-epoch study that includes concurrent mid-infrared and radio interferometry of an oxygen-rich Mira star.

Methods. We obtained mid-infrared interferometry of S Ori with VLTI/MIDI at four epochs in December 2004, February/March 2005, November 2005, and December 2005. We concurrently observed $v=1, J=1-0$ (43.1 GHz), and $v=2, J=1-0(42.8 \mathrm{GHz})$ $\mathrm{SiO}$ maser emission toward S Ori with the VLBA in January, February, and November 2005. The MIDI data are analyzed using self-excited dynamic model atmospheres including molecular layers, complemented by a radiative transfer model of the circumstellar dust shell. The VLBA data are reduced to the spatial structure and kinematics of the maser spots.

Results. The modeling of our MIDI data results in phase-dependent continuum photospheric angular diameters of $9.0 \pm 0.3$ mas (phase 0.42), 7.9 \pm 0.1 mas (0.55), $9.7 \pm 0.1$ mas (1.16), and $9.5 \pm 0.4$ mas (1.27). The dust shell can best be modeled with $\mathrm{Al}_{2} \mathrm{O}_{3}$ grains using phase-dependent inner boundary radii between 1.8 and 2.4 photospheric radii. The dust shell appears to be more compact with greater optical depth near visual minimum $\left(\tau_{V} \sim 2.5\right)$, and more extended with lower optical depth after visual maximum $\left(\tau_{V} \sim 1.5\right)$. The ratios of the $43.1 \mathrm{GHz} / 42.8 \mathrm{GHz} \mathrm{SiO}$ maser ring radii to the photospheric radii are $2.2 \pm 0.3 / 2.1 \pm 0.2$ (phase 0.44 ), $2.4 \pm 0.3 / 2.3 \pm 0.4(0.55)$, and $2.1 \pm 0.3 / 1.9 \pm 0.2(1.15)$. The maser spots mark the region of the molecular atmospheric layers just beyond the steepest decrease in the mid-infrared model intensity profile. Their velocity structure indicates a radial gas expansion. Conclusions. S Ori shows significant phase-dependences of photospheric radii and dust shell parameters. $\mathrm{Al}_{2} \mathrm{O}_{3}$ dust grains and $\mathrm{SiO}$ maser spots form at relatively small radii of $\sim 1.8-2.4$ photospheric radii. Our results suggest increased mass loss and dust formation close to the surface near the minimum visual phase, when $\mathrm{Al}_{2} \mathrm{O}_{3}$ dust grains are co-located with the molecular gas and the $\mathrm{SiO}$ maser shells, and a more expanded dust shell after visual maximum. Silicon does not appear to be bound in dust, as our data show no sign of silicate grains.
\end{abstract}

Key words. techniques: interferometric - masers - stars: AGB and post-AGB - stars: atmospheres - stars: mass-loss stars: individual: S Orionis

\section{Introduction}

The evolution of cool luminous stars, including Mira variables, is accompanied by significant mass loss to the circumstellar environment (CSE) with mass-loss rates of up to $10^{-4} M_{\odot} /$ year

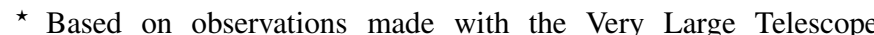
Interferometer (VLTI) at the Paranal Observatory under program IDs 074.D-0075 and 076.D-0338.

$\star \star$ Based on observations made with the Very Long Baseline Array (VLBA) under project BB192. The VLBA is operated by the National Radio Astronomy Observatory (NRAO). The National Radio Astronomy Observatory is a facility of the National Science Foundation operated under cooperative agreement by Associate Universities, Inc.

$\star \star \star$ Tables A.1-A.4 are only available in electronic form at the CDS via anonymous ftp to cdsarc.u-strasbg.fr (130.79.128.5) or via http://cdsweb.u-strasbg.fr/cgi-bin/qcat?J/A+A/470/191

Color versions of Figs. 2-8, 10, and 11 are available in electronic form via http: //www. aanda.org (e.g., Jura \& Kleinmann 1990). This mass-loss process significantly affects any further stellar evolution and is one of the most important sources for chemical enrichment of the interstellar medium. The detailed nature of the mass-loss process from evolved stars, and especially its connection with the pulsation mechanism in the case of Mira variable stars, is a matter of current investigation.

The conditions close to the stellar surface can be studied well by means of optical long-baseline interferometry. This technique has provided information regarding the stellar photospheric diameter, effective temperature, center-to-limb intensity variation (CLV), and atmospheric molecular layers for a number of Mira variables (see, e.g., Quirrenbach et al. 1992; Haniff et al. 1995; van Belle et al. 1996; Thompson et al. 2002; Mennesson et al. 2002; Hofmann et al. 2002; van Belle et al. 2002; Perrin et al. 2004; Millan-Gabet et al. 2005).

Woodruff et al. (2004) and Fedele et al. (2005) have recently shown that observed near-infrared $K$-band visibilities of 
the oxygen-rich prototype Mira variables $o$ Cet and R Leo are very different from uniform disc (UD) models already in the first lobe of the visibility function, and that they correspond closely to predictions by self-excited dynamic Mira model atmospheres that include effects from molecular layers (models by Hofmann et al. 1998; Tej et al. 2003b; Ireland et al. 2004a,b). Ohnaka et al. (2006b) studied the comparison of the same dynamic Mira model atmospheres with mid-infrared interferometric and spectroscopic observations. Recently, Ireland \& Scholz (2006) added the formation of dust in a self-consistent way to the same dynamic Mira model atmospheres. They find that dust would form at 2-3 times the average photospheric radius for certain plausible parameter values.

The structure of the atmospheric molecular shells located above the photosphere, as well as the dust shell, can be probed by mid-infrared interferometry. This has been successfully demonstrated by Ohnaka et al. (2005) using the spectro-interferometric capabilities of the VLTI/MIDI facility for observations of the oxygen-rich Mira star RR Sco. The model used in this work includes a warm molecular layer consisting of $\mathrm{SiO}$ and $\mathrm{H}_{2} \mathrm{O}$, as well as an optically-thin dust shell of $\mathrm{Al}_{2} \mathrm{O}_{3}$ and silicate. Recent VLTI/MIDI observations of the carbon-rich Mira star V Oph by Ohnaka et al. (2007) indicate that carbon-rich Miras also have extended atmospheric layers of polyatomic molecules $\left(\mathrm{C}_{2} \mathrm{H}_{2}\right)$ and dust shells (amorphous carbon and $\mathrm{SiC}$ ). Information on dust shells around Mira variables has also been derived using mid-infrared interferometry with the Berkeley Infrared Spatial Interferometer ISI (see e.g., Danchi et al. 1994; Weiner et al. 2006; Tatebe et al. 2006). Ireland et al. (2005) recently used $0.9 \mu \mathrm{m}$ interferometric polarimetry measurements of the Mira variables R Car and RR Sco to place constraints on the distribution of the dust shell.

Complementary information regarding the molecular shells around oxygen-rich AGB stars can be obtained by observing the maser radiation that some of these molecules emit. Maser emission from the three most common maser molecules, $\mathrm{SiO}, \mathrm{H}_{2} \mathrm{O}$, and $\mathrm{OH}$, traces regions of the CSE on scales from a few to several hundred AU. Masers provide a unique probe of structure, kinematics, and polarization properties of the environment of these stars. The $\mathrm{SiO}$ maser radiation in the CSE of oxygen-rich Mira variables has been mapped using the Very Long Baseline Array (VLBA) by, e.g., Diamond et al. (1994), Kemball \& Diamond (1997), Boboltz et al. (1997), Diamond \& Kemball (2003), Cotton et al. (2004), Boboltz \& Wittkowski (2005, hereafter BW05), Boboltz \& Diamond (2005), and Cotton et al. (2006). The $\mathrm{SiO}$ maser emission is typically found to arise from a clumpy ring within a few stellar radii of the photosphere, indicating a tangential amplification process. Humphreys et al. $(1996,2002)$ have predicted the location at which $\mathrm{SiO}$ molecules form in the CSE of Mira variables by combining maser models with hydrodynamic pulsation models. Their model predictions are roughly consistent with the measurements mentioned above.

Observational results regarding the detailed relationships between the stellar photosphere, the molecular layer, the dust shell, and the $\mathrm{SiO}$ maser ring often suffer from uncertainties inherent in comparing observations of variable stars widely separated in time and stellar phase, as discussed in BW05. To overcome this limitation, we have established a program of concurrent infrared interferometry using the VLTI and radio interferometry at the VLBA. The former aims at constraining the photospheric radius, the characteristics of atmospheric molecular layers, and parameters of the dust shell. The latter aims at concurrently mapping the $\mathrm{SiO}$ maser emission. Our final goal is a better understanding of the mass-loss process and its connection to stellar pulsation. Our pilot study in BW05 on the Mira variable S Ori included coordinated near-infrared $K$-band interferometry to constrain the stellar photospheric diameter and VLBA mapping of the $\mathrm{SiO}$ maser radiation toward this source.

A further uncertainty in comparing photospheric radii to the extensions of the dust and maser shells arises from the complication that near- and mid-infrared CLVs of finite bandwidth include a blend of intensities from continuum-forming layers and overlying molecular layers. This effect has often resulted in overestimated continuum photospheric diameter values (see, e.g. the discussions in Jacob \& Scholz 2002; Mennesson et al. 2002; van Belle et al. 2002; Woodruff et al. 2004; Ireland et al. 2004a,b; Perrin et al. 2004; Fedele et al. 2005). An overestimated photospheric diameter would result in biased relative distances of the dust shell and the maser ring from the stellar surface, even if obtained at the same stellar phase and cycle. A detailed comparison of observations to dynamic model atmospheres, as mentioned above, can be used to relate the observable quantities to the continuum photospheric radius and thus to overcome this limitation.

Here, we present VLTI/MIDI mid-infrared interferometry of S Ori at 4 epochs/stellar phases and coordinated VLBA mapping of the $\mathrm{SiO} 42.8 \mathrm{GHz}$ and $43.1 \mathrm{GHz}$ maser transitions at 3 epochs/stellar phases that are contemporaneous to the first 3 of our 4 MIDI epochs. The first two epochs are located near the stellar minimum, and the later epochs shortly after the following stellar maximum.

\section{Lightcurve and characteristics of S Ori}

$\mathrm{S}$ Ori is a Mira variable star with spectral type M6.5e-M9.5e and $V$ magnitude 7.2-14.0 (Samus et al. 2004). Templeton et al. (2005) report that S Ori's period appears to vary in a seemingly sinusoidal fashion between about 400 and 450 days over about the past 100 years. The mean period is $\bar{P}=420.0$ days with a latest period, corresponding to our observation epochs, of $P \approx 430$ days. We find a good correspondence with the AAVSO (Henden et al. 2006) and AFOEV (CDS) data for the cycle of our observations, as well as for the most recent $\sim 5$ cycles using $P=430$ days and a Julian Date of last maximum brightness $T_{0}=2453190$ days. Figure 1 shows the visual lightcurve of $\mathrm{S}$ Ori as a function of Julian Date and stellar phase based on these values. Also indicated are the dates/epochs of our VLTI and VLBA observations as described in Sects. 3 and 4.

The distance to S Ori, as for many Mira stars, is not well known. Van Belle et al. (2002) have estimated the distance to S Ori to $480 \mathrm{pc} \pm 120 \mathrm{pc}$ based on a calibration of the periodluminosity relationship by Feast et al. (1989), and we adopt this value.

The near-infrared $K$-band UD angular diameter $\Theta_{\mathrm{UD}}^{K}$ of S Ori has been measured by van Belle et al. (1996), Millan-Gabet et al. (2005), and by BW05 to values between 9.6 mas and 10.5 mas at different phases. Diameter measurements of S Ori at other wavelengths have so far not been reported.

$\mathrm{S}$ Ori exhibits $\mathrm{SiO}$ and $\mathrm{OH}$ maser emission (Benson et al. 1990), while a detection of $\mathrm{H}_{2} \mathrm{O}$ maser toward $\mathrm{S}$ Ori has not been reported. The maps of the $\mathrm{SiO}$ maser emission in BW05 are the first toward S Ori. They derived average distances and standard deviations of the $\mathrm{SiO}$ maser spots from the center of their distribution at phase 0.73 of $9.4 \pm 1.4$ mas and $8.8 \pm 1.7$ mas for the $43.1 \mathrm{GHz}$ and $42.8 \mathrm{GHz}$ transitions, respectively.

Sloan \& Price (1998) report on a relatively low dustemission coefficient (Dec, the total emission of the dust to the total emission of the star in the wavelength range 7.7-14.0 $\mu \mathrm{m}$ ) 
Table 1. VLTI/MIDI observations of S Ori.

\begin{tabular}{lllrlllllrrrr}
\hline \hline Ep. & Date & $\begin{array}{r}\text { Time } \\
\text { UTC }\end{array}$ & $\begin{array}{r}\text { JD } \\
-2.4 \mathrm{E6}\end{array}$ & $\Phi_{\text {vis }}$ & Conf. & $B$ & $\begin{array}{l}\text { Disp. } \\
\text { Elem. }\end{array}$ & BC & $\begin{array}{r}B_{\mathrm{p}} \\
{[\mathrm{m}]}\end{array}$ & $\begin{array}{r}\text { PA } \\
{[\mathrm{deg}]}\end{array}$ & $\begin{array}{r}\text { Seeing } \\
{\left[{ }^{\prime \prime}\right]}\end{array}$ & $\begin{array}{r}\tau_{0} \\
{[\mathrm{msec}]}\end{array}$ \\
\hline A & $2004-12-31$ & $02: 43$ & 53370.6 & 0.42 & UT3-UT4 & $62 \mathrm{~m}$ & Prism & HS & 62.27 & -71.9 & 0.76 & 3.4 \\
B & $2005-02-21$ & $02: 37$ & 53422.6 & 0.54 & UT2-UT4 & $89 \mathrm{~m}$ & Prism & HS & 74.31 & 83.0 & 0.74 & 3.6 \\
B & $2005-03-01$ & $02: 39$ & 53430.6 & 0.56 & UT3-UT4 & $62 \mathrm{~m}$ & Prism & HS & 41.65 & -55.6 & 0.62 & 3.6 \\
B & $2005-03-03$ & $00: 59$ & 53432.5 & 0.56 & UT2-UT4 & $89 \mathrm{~m}$ & Prism & HS & 84.35 & 82.7 & 0.98 & 3.7 \\
C & $2005-11-12$ & $08: 57$ & 53686.9 & 1.16 & UT1-UT4 & $130 \mathrm{~m}$ & Prism & SP & 123.02 & 62.8 & 0.54 & 4.4 \\
C & $2005-11-15$ & $07: 21$ & 53689.8 & 1.16 & UT2-UT3 & $47 \mathrm{~m}$ & Prism & SP & 46.26 & 43.9 & 0.61 & 2.9 \\
D & $2005-12-30$ & $03: 00$ & 53734.6 & 1.27 & UT1-UT4 & $130 \mathrm{~m}$ & Prism & SP & 123.89 & 59.4 & 1.12 & 2.8 \\
\hline
\end{tabular}
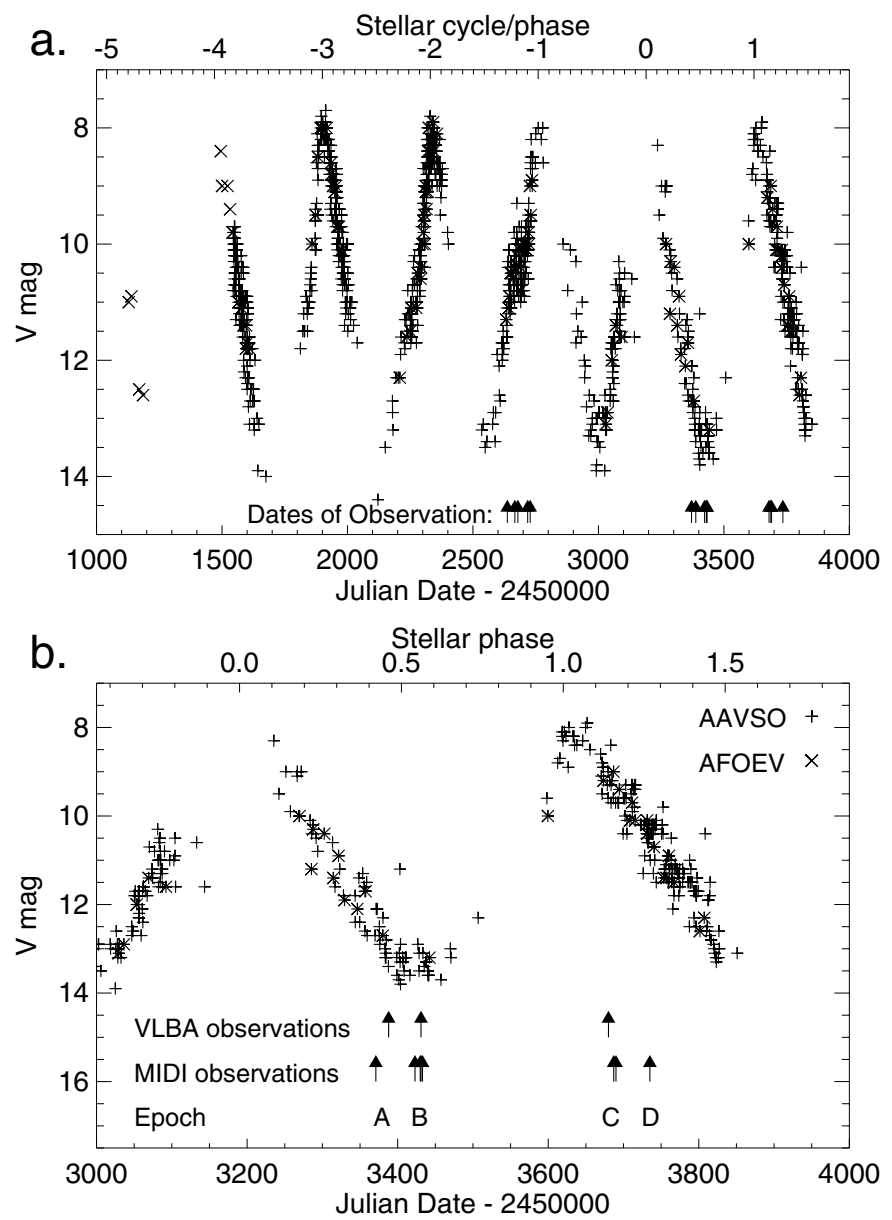

Fig. 1. Visual lightcurve of S Ori as a function of Julian Date and stellar cycle/phase. Data are from the AAVSO (Henden et al. 2006) and AFOEV (CDS) databases. We adopt a period of $P=430$ days and JD of last maximum brightness $T_{0}=2453190$. The arrows indicate the dates of our VLTI and VLBA observations. a) The most recent 5-6 cycles illustrating the correspondence of the data with our adopted $P$ and $T_{0}$ values. The indicated dates of observations are the epochs from BW05 (JD between 2600 and 2800) and the epochs of the present paper (JD between 3300 and 3800). b) Enlarged view covering our VLTI/MIDI and VLBA observations presented in this paper. The dates of observations are combined into 4 epochs A, B, C, D.

for S Ori of 0.24, i.e. similar to RR Sco $(\mathrm{Dec}=0.21)$ or R Leo $($ Dec $=0.23)$, indicating an optically thin dust shell.

\section{VLTI/MIDI measurements}

We obtained mid-infrared $(8-13 \mu \mathrm{m})$ interferometry of S Ori with the instrument MIDI (Leinert et al. 2003) at the ESO VLT
Interferometer (VLTI; Glindemann et al. 2003) in service mode between 31 December 2004 and 30 December 2005. Light was combined from two $8 \mathrm{~m}$ Unit Telescopes (UTs). We chose to use the prism with spectral resolution $R=\lambda / \Delta \lambda=30$ to disperse the interferograms. Two various techniques of beam combinations were used, namely HIGH_SENS (HS, 2004-12-31 to 2005-03-03) and SCI_PHOT (SP, 2005-11-12 to 2005-12-30) combinations. Using the HS combination, all arriving light is first combined interferometrically, and the photometric spectrum for each incoming beam is recorded sequentially. Using the SP combination, beam splitters are used to record the interferograms and the photometric spectra simultaneously. An estimate of the photometric count rates at the time of recording the interferometric data is needed to compute the raw visibility values, and the SP technique is thus expected to result in a higher precision of the visibility measurement for bright targets. Our observations followed the standard procedures for MIDI service mode observations as described in detail in the MIDI user manual ${ }^{1}$. For principles of observations with MIDI see also Leinert et al. (2004).

The details of our observations are listed in Table 1. Listed are the epoch, date, time, and Julian Date (JD) of the observation; the visual phase $\Phi_{\text {vis }}$; the baseline configuration used with its ground length $B$; the dispersive element and beam combiner BC (HS for High_Sens and SP for Sci_Phot); and the projected baseline length $B_{\mathrm{p}}$ and its position angle PA on sky (deg. east of north). The last two columns describe the ambient conditions DIMM seeing, and coherence time $\tau_{0}$, both at $500 \mathrm{~nm}$. Dates of observation were combined into 4 epochs A, B, C, D. Each epoch has a width of less than 17 days, i.e. less than $5 \%$ of S Ori's variability period. The dates of observation and the epochs compared to the visual lightcurve are indicated in Fig. 1. We estimate the uncertainty in assigning a visual phase value to each of our observing epochs to $\sim 0.1$, taking into account both the time widths of our epochs and the uncertainty in the choice of S Ori's current variability period and date of last maximum (see Sect. 2). Epochs A and B are located around stellar minimum with a separation of about 2 months (phase difference $\sim 0.1$ ), while epochs $C$ and D occurred about a year later shortly after the following stellar maximum with a separation of 6 weeks. Additional MIDI/UT data on S Ori from 2004-1229, 2005-01-02, 2005-02-28, 2006-03-12, 2006-03-12, as well as MIDI/AT data taken between October 2005 and March 2006 were not used because of various technical problems with these data sets. The interferometric field of view of MIDI used with the UTs is $\sim 250$ mas, which corresponds to about 25 times the expected photospheric angular diameter of S Ori.

We used $\theta$ CMa (HD 50778) as the interferometric calibrator, a K4 giant at a distance on sky of $27^{\circ}$, which was observed within about $30 \mathrm{~min}$ of each S Ori observation. A number of

\footnotetext{
${ }^{1}$ http://www.eso.org/instruments/midi
} 
Table 2. Properties of the MIDI calibration stars used.

\begin{tabular}{rrrcrrr}
\hline \hline HD & Name & Sp.T. & $\begin{array}{c}\Theta_{\mathrm{LD}} \\
{[\mathrm{mas}]}\end{array}$ & $\begin{array}{r}T_{\text {eff }} \\
{[\mathrm{K}]}\end{array}$ & $\begin{array}{r}F_{12} \\
{[\mathrm{Jy}]}\end{array}$ & $\begin{array}{r}\zeta \\
{\left[{ }^{\circ}\right]}\end{array}$ \\
\hline 50778 & $\theta$ CMa & K4 III & $3.90 \pm 0.02$ & 4049 & 24.6 & 27 \\
31421 & $o^{2}$ Ori & K2 III & $2.65 \pm 0.01$ & 4500 & 13.6 & 12 \\
49161 & 17 Mon & K4 III & $2.44 \pm 0.01$ & 4248 & 7.2 & 20 \\
61935 & $\alpha$ Mon & G9 III & $2.24 \pm 0.01$ & 4813 & 10.2 & 36 \\
94510 & u Car & K1 III & $2.23 \pm 0.01$ & 4951 & 6.9 & 90 \\
107446 & $\epsilon$ Cru & K3.5 III & $4.43 \pm 0.02$ & 4139 & 32.4 & 101 \\
\hline
\end{tabular}

observations of other MIDI calibration stars using the same instrument settings were obtained during some of our observation nights for other programs, and we make use of these data as well. Table 2 lists the calibration stars and their properties. These parameters are taken from the instrument consortium's catalog ${ }^{2}$. Listed are the HD number, the name, the spectral type, the limbdarkened diameter $\Theta_{\mathrm{LD}}$, the effective temperature $T_{\text {eff }}$, the flux at $12 \mu \mathrm{m}$, and the distance on sky from S Ori $\zeta$. The UD diameter for $\mathrm{K}$ giants in the mid-infrared based on spherical model atmospheres is smaller by $\sim 5 \%$ than $\Theta_{\mathrm{LD}}$ (estimated with procedures as used in Wittkowski et al. 2006). HD 50778 is our main calibrator. The other calibrators were observed during some of our observation nights for other programs. Among the interferometric calibration stars listed in Table 2, absolutely calibrated spectra are available for HD 31421 and HD 61935 (Cohen et al. 1999).

\subsection{MIDI data reduction}

Mean coherence factors and photometric count rates were obtained for each data set of S Ori and the calibration stars using the MIA+EWS software package, version 1.4 (Jaffe, Koehler, Cotton, Chesneau, et $\mathrm{al}^{3}$ ), complemented by the tool MyMidiGui, version $1.41\left(\mathrm{Hummel}^{4}\right)$. Data for 32 spectral channels within the nominal MIDI wavelength range from $8.0 \mu \mathrm{m}$ to $13.0 \mu \mathrm{m}$ were used. The detector masks were calculated by the procedure of MyMidiGui, and were used for both the MIA and the EWS analysis. The results obtained by MIA and EWS were found to be virtually identical for all datasets. We chose to use in the following the results based on the EWS analysis. It is based on a coherent integration of the fringe signal after numerical compensation of optical path differences in each scan.

Calibrated visibility spectra for S Ori were derived using the transfer function obtained for our calibrator HD 50778. The scatter of all obtained transfer functions during the night was used to estimate its uncertainty. The latter depends on the sample of calibrations stars available per night, and we assumed a minimum uncertainty of the transfer function of 0.1 based on nights when many values were obtained. The total error of our calibrated S Ori visibility spectrum includes the errors of the raw coherence factors computed by EWS, the uncertainty of the calibrator diameters, and the scatter of the transfer function during the night. The total error is dominated by systematic uncertainties for the height of the interferometric transfer function, so that the relative accuracy of the visibility values among the spectral channels is better than indicated by our total error bars.

We absolutely calibrated the photometric spectrum of S Ori for each data set using the interferometric calibrator HD 50778

\footnotetext{
2 http://www. ster. kuleuven.ac.be/ tijl/ MIDI_calibration/mcc.txt

3 http://www.strw. leidenuniv.nl/ koehler/MIDI

4 http://www. Sc. eso.org/ chummel/midi/midi.html
}

also as the photometric calibration star. The absolutely calibrated photometric spectrum of HD 50778 was not available directly. We used instead the average of the available spectra of HD 20644 and HD 87837 (Cohen et al. 1999), scaled with the IRAS $12 \mu \mathrm{m}$ flux (Beichmann et al. 1988) to the level of HD 50778. These stars have the same spectral type (K4 III) and very similar effective temperatures as HD 50778 (4058 K and $4094 \mathrm{~K}$ versus $4049 \mathrm{~K}$ ). In addition, we calibrated the photometric spectrum of HD 50778 using the spectro-photometric calibrator HD 31421, which was observed close in time during the night 2005-03-03, and verified that our synthetic HD 50778 spectrum is valid. We obtained integrated $8.0-13.0 \mu \mathrm{m} \mathrm{S}$ Ori flux densities for our MIDI epochs A to D of $187 \pm 12 \mathrm{Jy}, 152 \pm 17 \mathrm{Jy}$, $198 \pm 18 \mathrm{Jy}$, and $203 \pm 13 \mathrm{Jy}$. For comparison, the integrated IRAS LRS flux density for the same bandpass $(8.0-13.0 \mu \mathrm{m})$ is $169 \mathrm{Jy}$.

The MIDI calibrated visibility, as well as calibrated flux values, are detailed in Table A.1.

\subsection{MIDI data reduction results}

As an alternative and probably more intuitive quantity compared to the visibility values, we calculated the equivalent UD diameters. The equivalent UD diameter was computed for each projected baseline length and spectral channel separately and defined as the diameter of that uniform disc that results in the same visibility value at each separate data point. Note that the true intensity profile is not expected to be a uniform disc. As a result, the equivalent UD diameters for different projected baseline lengths are not expected to coincide (not even for a circularly symmetric intensity profile). However, these values are fully equivalent to the calibrated visibility values and can provide a first rough estimate of the characteristic size of the target.

Figures 2-5 show, for MIDI epochs A-D, respectively, the obtained MIDI flux spectra, the MIDI visibility values as a function of wavelength, the MIDI visibility values as a function of spatial frequency for the example of three spectral channels and the equivalent UD diameter values. Also shown are the synthetic visibility and flux values of the atmosphere and dust models described below in Sect. 3.3 in each panel, as well as the intensity profiles of these models for the example of three bandpasses in the bottom panels.

The general shape of our $\mathrm{S}$ Ori visibility functions, or equivalent UD diameter values, in Figs. 2-5 is qualitatively similar to the MIDI data of the Mira variable RR Sco described in Ohnaka et al. (2005). Objects such as these are characterized by a partially resolved stellar disc, including atmospheric molecular layers that are optically thick at mid-infrared wavelengths, surrounded by a spatially resolved, optically thin dust shell. The visibility increases between about $8-9 \mu \mathrm{m}$, corresponding to a quasi-constant equivalent UD diameter that is roughly twice the continuum photospheric size. Here, the observed intensity is dominated by radiation from optically thick molecular layers at distances up to roughly two continuum photospheric radii. Beyond about $9-10 \mu \mathrm{m}$, the visibility function flattens, corresponding to an increasing equivalent UD diameter. In this wavelength region, spatially resolved, optically thin radiation from the dust shell starts to represent a considerable part of the total intensity. In addition, extinction of the stellar light by the dust shell becomes important. At the longest wavelengths of MIDI, spatially resolved radiation from the dust shell dominates the measured intensity. A detailed model of S Ori's atmosphere and dust shell is described in Sects. 3.3 and 3.4. 

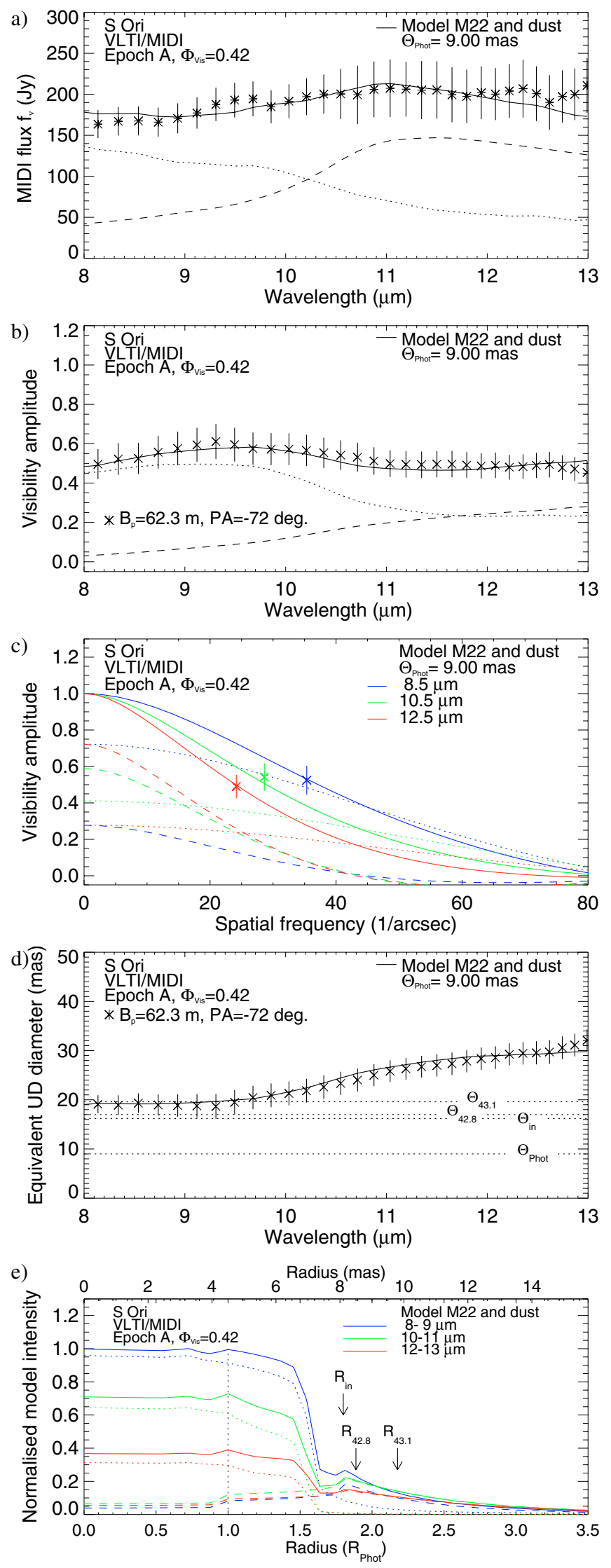

\subsection{MIDI data modeling}

Mid-infrared photometric and interferometric data of Mira stars are in general sensitive to details of the structure of the stellar
Fig. 2. Results of our VLTI/MIDI $8-13 \mu \mathrm{m}$ interferometry of $\mathrm{S}$ Ori at epoch A, stellar phase 0.42 . Shown are our MIDI data compared to the best-fitting model with parameters listed in Table 3. In each panel, the $\mathrm{x}$-symbols with error bars denote the measured values, the solid lines the synthetic values of the overall model consisting of the stellar model atmosphere and the dust shell. The dotted lines denote the contribution from the attenuated atmosphere model alone, and the dashed lines the contribution of only the dust shell (attenuated input radiation subtracted). a) The calibrated MIDI flux spectrum. b) The calibrated MIDI visibility values as a function of wavelength. c) The calibrated MIDI visibility values as a function of spatial frequency for the example of three spectral channels at wavelengths $8.5 \mu \mathrm{m}, 10.5 \mu \mathrm{m}$, and $12.5 \mu \mathrm{m}$. d) The equivalent UD diameter values. Also indicated are the photospheric angular diameter $\Theta_{\text {Phot }}$ and the inner dust shell boundary $\Theta_{\text {in }}$ as obtained by our model fit, as well as the mean $42.8 \mathrm{GHz}$ and $43.1 \mathrm{GHz}$ $\mathrm{SiO}$ ring angular diameters (Sect. 4). e) The model intensity profiles at spectral bands $8-9 \mu \mathrm{m}, 10-11 \mu \mathrm{m}$, and $12-13 \mu \mathrm{m}$. The bottom abscissa shows the radius as a function of the photospheric radius $R_{\text {Phot }}$, and the top abscissa the corresponding angular scale obtained from our model fit. The arrows indicate the location of the inner boundary radius of the dust shell $R_{\text {in }}$, as well as of the mean $43.1 \mathrm{GHz}$ and $42.8 \mathrm{GHz} \mathrm{SiO}$ maser spot radii $R_{43.1}$ and $R_{42.8}$. atmosphere in which molecular layers of various geometrical and optical characteristics (e.g., Tej et al. 2003a) lie above the continuum-forming photosphere, as well as to the surrounding dust shell (cf. e.g., Ohnaka et al. 2005, 2006b; 

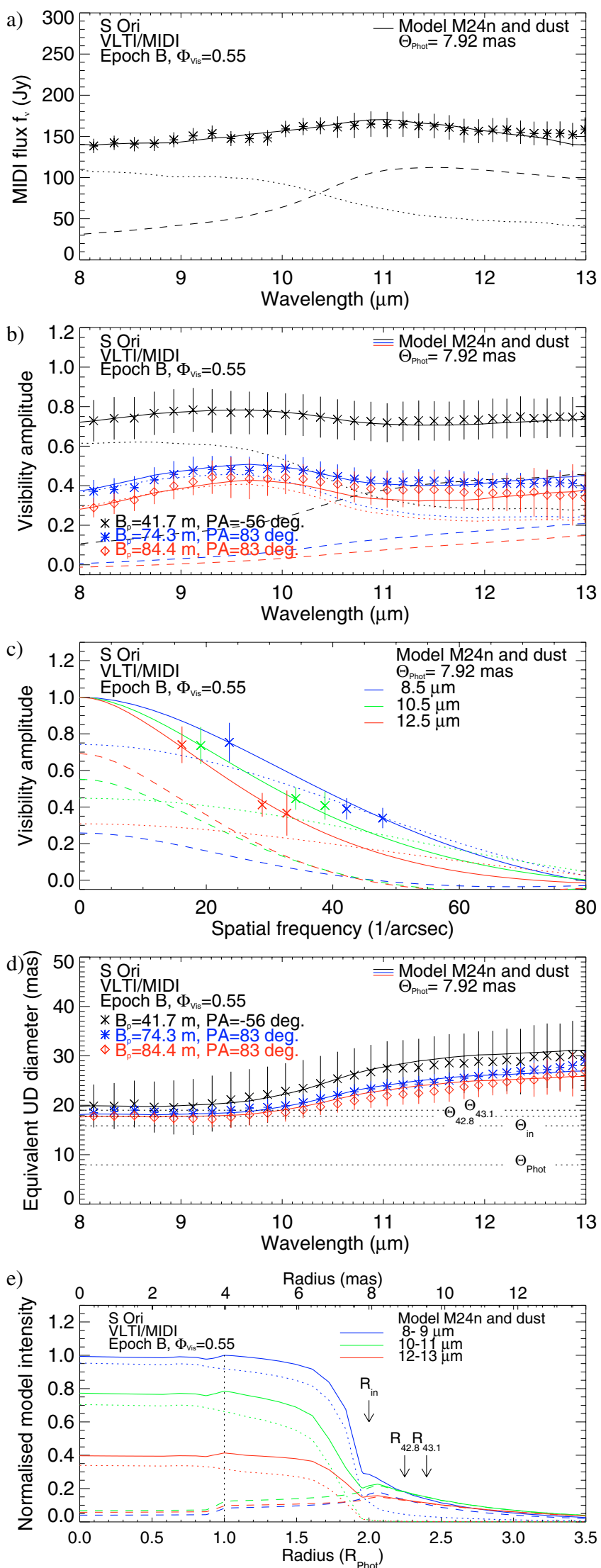

Fig. 3. Results of our VLTI/MIDI $8-13 \mu \mathrm{m}$ interferometry of S Ori at epoch B, stellar phase 0.55. As Fig. 2 .
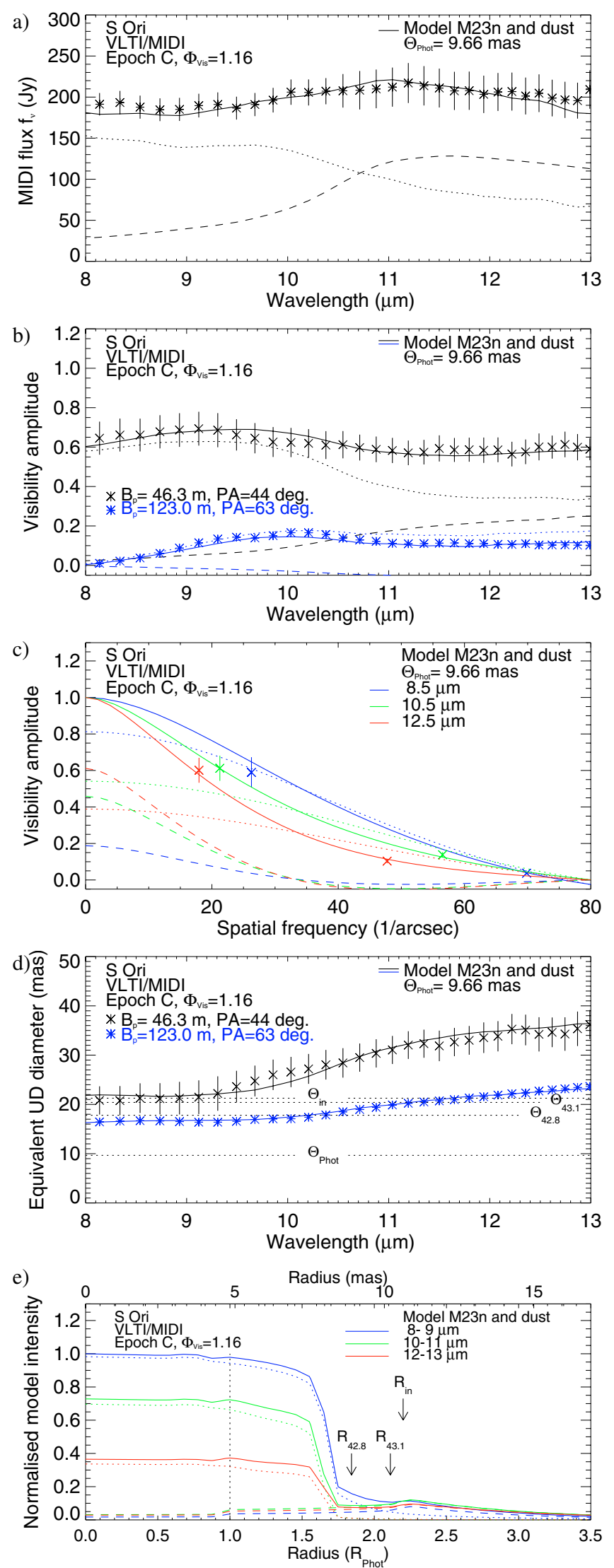

Fig. 4. Results of our VLTI/MIDI 8-13 $\mu \mathrm{m}$ interferometry of S Ori at epoch C, stellar phase 1.16. As Fig. 2. 

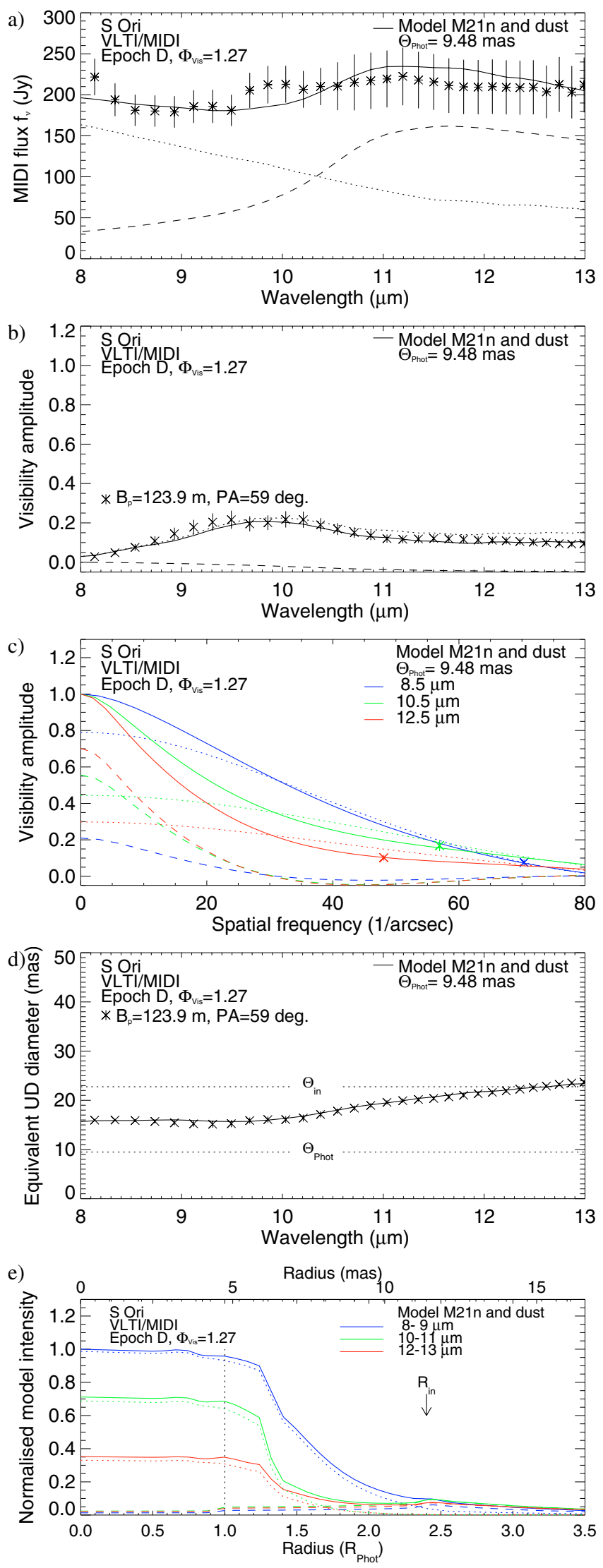

Fig. 5. Results of our VLTI/MIDI $8-13 \mu \mathrm{m}$ interferometry of S Ori at epoch D, stellar phase 1.27. As Fig. 2.

Ireland \& Scholz 2006; Weiner et al. 2006). We used the P and $\mathrm{M}$ model atmosphere series (Ireland et al. 2004a,b) to describe the stellar atmosphere including the continuum photosphere and overlying molecular layers, and added an ad-hoc radiative transfer model of the dust shell. The details of our modeling are described in the following.

\subsubsection{Atmosphere model for S Ori}

Few dynamic atmosphere models for oxygen-rich Mira stars are available that include the effects of molecular layers (see, e.g., Scholz 2001, 2003). The P and M model series (Ireland et al. 2004a,b) are complete self-excited dynamic model atmosphere of Mira stars, and they have successfully explained a lot of observational data (e.g.; Hofmann et al. 2000, 2001, 2002; Woodruff et al. 2004; Fedele et al. 2005). These model series have been constructed to match the prototype oxygen-rich Mira stars $o$ Cet and R Leo. The $\mathrm{P}$ and $\mathrm{M}$ series differ with respect to the mass of the so-called "parent star", which is the hypothetical non-pulsating equivalent of the pulsating Mira variable. The geometric pulsation of the Mira occurs around the parent star's Rosseland radius $R=R\left(\tau_{\text {Ross }}=1\right)$ (Ireland et al. 2004a,b). The parent star has solar metallicity, luminosity $L / L_{\odot}=3470$, period 332 days, mass $M / M_{\odot}=1.0$ (P series) and $1.2(\mathrm{M})$, radius $R_{\mathrm{p}} / R_{\odot}=241(\mathrm{P})$ and $260(\mathrm{M})$ (Hofmann et al. 1998). The moderately larger radius of the $\mathrm{M}$-series parent star leads to a slightly lower effective temperature for the parent star and systematically lower phase-dependent effective temperatures for the pulsating Mira compared to the $\mathrm{P}$ series. The $\mathrm{M}$ models tend to exhibit less pronounced cycle-to-cycle variations than the $\mathrm{P}$ models and to have more compact atmospheres (Ireland et al. 2004b). Effects on interferometric diameter measurements resulting from the differences between the $\mathrm{P}$ and $\mathrm{M}$ model series are subtle. When comparing $\mathrm{P}$ and $\mathrm{M}$ models of similar phases to near-infrared interferometric data of $\mathrm{R}$ Leo, the resulting Rosseland angular diameter values were found to agree within $\lesssim 1.5 \%$ (Fedele et al. 2005).

Compared to $o$ Cet and R Leo, for which the P and M model series are designed, S Ori is a slightly cooler Mira variable (M6.5-M9.5 versus M5-M9/M6-M9.5, Samus et al. 2004) with a longer period $(420 \mathrm{~d}$ versus $332 \mathrm{~d} / 310 \mathrm{~d}$, Samus et al. 2004), a larger main sequence precursor mass $\left(1.3 M_{\odot}\right.$ versus $1.0 M_{\odot} / 1.2 M_{\odot}$, Wyatt \& Cahn 1983), and a larger radius (mean continuum photospheric radii roughly $R \sim 450 R_{\odot}$ versus $\sim 350 R_{\odot}$; based on BW05, Woodruff et al. 2004; Fedele et al. 2005). However, when scaled to variability phases between 0 and 1 and to the corresponding angular size on sky, the general model results are not expected to be dramatically different for S Ori compared to Mira stars, such as $o$ Cet and R Leo. Hereby, the $\mathrm{M}$ series is expected to be better-suited to describing S Ori than the $\mathrm{P}$ series because of its higher parent-star mass. We concluded that the use of the $\mathrm{M}$ series is currently the best available option for describing S Ori's atmosphere including the continuum photosphere and overlying atmospheric molecular layers.

\subsubsection{Dust-shell model for S Ori}

Except for the dust-enshrouded very-long-period Mira IW Hya (Jeong et al. 2003), models that self-consistently combine detailed dynamic atmosphere models and the formation of dust shells are currently not readily available for oxygen-rich AGB stars. However, the first attempts in this direction have just been made (Ireland \& Scholz 2006, Woitke 2006).

Dust shells around AGB stars have often been modeled using ad-hoc radiative transfer calculations (see; for instance; Danchi et al. 1994; Lorenz-Martins \& Pompeia 2000; Ohnaka et al. 2005, 2006a), and we followed this approach as the only 
currently available option for describing S Ori's dust shell. We employed the Monte Carlo radiative transfer code mcsim_mpi (Ohnaka et al. 2006a) to calculate the dust shell model of S Ori. For the central radiation source of the radiative transfer model, we used the spectral energy distribution (SED) of the respective $\mathrm{M}$ model atmosphere for the wavelength range from $0.32 \mu \mathrm{m}$ to $23 \mu \mathrm{m}$ and extended it by a blackbody approximation for longer wavelengths. Dust temperatures were calculated in radiative equilibrium.

Our MIDI spectra of S Ori do not exhibit any prominent silicate feature, indicating that silicate grains are not a major constituent of the dust shell. Lorenz-Martins \& Pompeia (2000) have investigated the envelopes of 31 oxygen-rich AGB stars using the IRAS LRS spectra. They classify the objects into three groups, whose IRAS LRS spectra can be modeled using either silicate grains, $\mathrm{Al}_{2} \mathrm{O}_{3}$ grains, or a mix thereof. $\mathrm{S}$ Ori is part of their study and belongs to the group of objects that can be modeled well with $\mathrm{Al}_{2} \mathrm{O}_{3}$ grains alone. They show that a blend of alumina $\mathrm{Al}_{2} \mathrm{O}_{3}$ grains (Koike et al. 1995) for $\lambda<8 \mu \mathrm{m}$ and porous amorphous $\mathrm{Al}_{2} \mathrm{O}_{3}$ grains (Begemann et al. 1997) for $\lambda>8 \mu \mathrm{m}$ gives the best agreement with the IRAS spectra, so we followed this approach (as in Ohnaka et al. 2005 for the case of RR Sco). Likewise, we considered small amounts of warm silicates (Ossenkopf et al. 1992) following the same works. The grain size was set to $0.1 \mu \mathrm{m}$ for both $\mathrm{Al}_{2} \mathrm{O}_{3}$ and silicates. We used the same inner boundary radius and density distribution for both dust species. The amount of dust of each species was characterized by the optical depths at a wavelength of $0.55 \mu \mathrm{m}$, denoted $\tau_{V}\left(\mathrm{Al}_{2} \mathrm{O}_{3}\right)$ and $\tau_{V}$ (silicate). The density distribution $\rho(r)$ was described by a single power law with index $p\left(\rho(r) \propto r^{-p}\right)$. We used the inner boundary radii in units of the stellar photospheric radius $R_{\text {Phot }}$. All radiative transfer models of the dust shell were calculated with an outer radius, so that the shell thickness is $R_{\text {out }} / R_{\text {in }}=1000$.

\subsubsection{The main model parameters}

The stellar atmospheric structure including the effects from atmospheric molecular layers is fully described by the choice of the specific model of the $M$ series. The $M$ series consists of 20 models for different phase and cycle combinations as detailed in Ireland et al. (2004b). Here, we used a grid of the six M models of cycle 2 of the series, which are M20 (model visual phase 0.05), M21n (0.10), M22 (0.25), M23n (0.30), M24n (0.40), and M25 (0.50). These models cover the range of our observations at phases 0.16 (epoch C), 0.27 (epoch D), 0.42 (epoch A), and 0.55 (epoch D). The absolute model phases with respect to observations are uncertain by at least 0.1 , with relative uncertainties of 0.01 to 0.02 between different models of the series (Ireland et al. 2004b). Likewise, the absolute assignment of visual phases to our epochs of observation is uncertain by about 0.1 (see Sect. 3). For each of these 6 atmosphere models, whose SEDs are used as central source, we calculated a grid of radiative transfer models of the dust shell. These grids included all combinations of optical depths $\tau_{V}\left(\mathrm{Al}_{2} \mathrm{O}_{3}\right)=1.0,1.5,2.0,2.5$, $3.0 ; \tau_{V}$ (silicate) $=0 \%, 10 \%$ of $\tau_{V}\left(\mathrm{Al}_{2} \mathrm{O}_{3}\right)$; inner boundary radii $R_{\text {in }} / R_{\text {Phot }}=1.8,2.0,2.2,2.4,2.6$; and gradients $p=2.0,2.5$, 3.0, 3.5, 4.0.

Any overall model was thus defined with this set of five independent parameters: (1) choice of the dust-free $\mathrm{M}$ model, (2) $\tau_{V}\left(\mathrm{Al}_{2} \mathrm{O}_{3}\right),(3) \tau_{V}$ (silicate), (4) $R_{\text {in }}$, (5) $p$. We used the welldefined continuum photospheric radius at $\lambda=1.04 \mu \mathrm{m} R_{1.04}$ of the respective dust-free $\mathrm{M}$ model to characterize the photospheric radius $R_{\text {Phot }}$; i.e. we set $R_{\text {Phot }}=R_{1.04}$. Any overall model is rigid; i.e. the distance of any model layer from $R_{\text {Phot }}$ is fixed with the parameters given above. The corresponding angular diameter $\Theta_{\text {Phot }}$ was treated as the only fit parameter when comparing any such overall model to our MIDI data.

\subsubsection{Synthetic visibility and flux values}

Stellar contribution Monochromatic CLVs of the M models were calculated at 3000 equally-spaced frequencies for the wavelength range $8 \mu \mathrm{m} \leq \lambda \leq 13 \mu \mathrm{m}$ as described in Ohnaka et al. (2006b), covering radii $r$ between 0 and $R_{\text {out }}^{\text {star }}=5 \times R_{\mathrm{p}}$. These CLVs were calculated with $\mathrm{H}_{2} \mathrm{O}$ and $\mathrm{SiO}(28 \mathrm{SiO}, 29 \mathrm{SiO}$, and $30 \mathrm{SiO}$ ) lines included. The line lists used for the calculations were the same as those used for modeling RR Sco by Ohnaka et al. (2005); and as in the case of RR Sco, we adopted silicon isotope ratios of $28 \mathrm{Si} / 29 \mathrm{Si}=15$ and $28 \mathrm{Si} / 30 \mathrm{Si}=20$. The $\mathrm{H}_{2} \mathrm{O}$ and $\mathrm{SiO}$ line opacities were computed assuming local thermodynamical equilibrium (LTE) and a Voigt profile with a microturbulent velocity of $3 \mathrm{~km} \mathrm{~s}^{-1}$.

The visibility values based on the stellar contribution were calculated for each spectral channel $c$ of MIDI as

$V_{c}^{\mathrm{star}}=\frac{\int_{v_{\min }(c)}^{v_{\max }(c)} \int_{0}^{R_{\text {out }}^{\mathrm{star}}} I_{v}^{\mathrm{star}}(r) \mathrm{e}^{-\tau(v)} J_{0}\left(\pi \Theta_{\text {out }}^{\mathrm{star}}(B / \lambda)\right) r \mathrm{~d} r \mathrm{~d} v}{\int_{v_{\min }(c)}^{v_{\max }(c)} \int_{0}^{R_{\text {out }}^{\mathrm{star}}} I_{\nu}^{\mathrm{star}}(r) \mathrm{e}^{-\tau(v)} r \mathrm{~d} r \mathrm{~d} v}$.

Here, $B$ is the projected baseline length used for the observation. Note that this computation is valid for the case of the EWS data reduction, which coherently integrates the fringe signal to directly obtain the visibility modulus. For the MIA analysis, which is a power spectrum analysis integrating the squared visibility modulus, the integration over frequency would need to be performed over the squared monochromatic visibility values (cf. Wittkowski et al. 2006). The observed stellar flux contribution $f_{c}^{\text {star }}$ was computed for each of MIDI's spectral channels as

$f_{c}^{\mathrm{star}}=2 \pi \frac{\Theta_{\mathrm{out}}^{\mathrm{star}}\left[{ }^{\prime \prime}\right] / 2}{206265} \int_{v_{\min }(c)}^{v_{\max }(c)} \int_{0}^{R_{\mathrm{out}}^{\mathrm{star}}} I_{v}^{\mathrm{star}}(r) \mathrm{e}^{-\tau(v)} r \mathrm{~d} r \mathrm{~d} v$.

The spectral channels of MIDI were assumed to have a rectangular sensitivity function between frequencies $v_{\min }$ and $v_{\max }$. The term $\mathrm{e}^{-\tau(v)}$ describes the extinction by the dust shell. The optical depth $\tau(v)$ was calculated based on tabulated absorption and scattering coefficients $Q_{\mathrm{abs}}$ and $Q_{\mathrm{sca}}$ of the two dust species $i$ as

$\tau(v)=\sum_{i} \tau^{i}\left(v_{0}\right) \frac{Q_{\mathrm{abs}}^{i}(v)+Q_{\mathrm{sca}}^{i}(v)}{Q_{\mathrm{abs}}^{i}\left(v_{0}\right)+Q_{\mathrm{sca}}^{i}\left(v_{0}\right)}$

where we used $v_{0}$ corresponding to $\lambda_{0}=0.55 \mu \mathrm{m}$.

Dust contribution Using the dust temperatures and monochromatic mean intensities computed by a Monte Carlo run with mcsim_mpi for a given set of dust shell parameters, we calculated monochromatic CLVs of the dust shell (excluding the contribution of the central star) with the ray-tracing method as described in Ohnaka et al. (2006a, 2007). The radii $r$ covered the range between 0 and $25 R_{\text {Phot }}$, and we denote the outermost model radius, where we set the intensity to 0 , by $R_{\text {out }}^{\text {dust }}=$ $25 \times R_{\text {Phot }}$. The outer radius of $25 R_{\text {Phot }}$ was chosen because the MIDI interferometric field of view of $\sim 250$ mas corresponds to 25 times the expected photospheric angular diameter of S Ori of $\sim 10$ mas. We verified that changing the outer model radius from $25 R_{\text {Phot }}$ to $50 R_{\text {Phot }}$ changed the diameter results by less than $0.3 \%$. This indicates that the contribution from dust to the overall CLV outside of 25 photospheric radii can be neglected 
Table 3. Fit results of our MIDI measurements of S Ori.

\begin{tabular}{lrrrr}
\hline \hline Epoch $\left(\Phi_{\text {Vis }}\right)$ & $\mathrm{A}(0.42)$ & $\mathrm{B}(0.55)$ & $\mathrm{C}(1.16)$ & $\mathrm{D}(1.27)$ \\
\hline Best model & $\mathrm{M} 22$ & $\mathrm{M} 24 \mathrm{n}$ & $\mathrm{M} 23 \mathrm{n}$ & $\mathrm{M} 21 \mathrm{n}$ \\
$\Phi_{\text {Model }}$ & 0.25 & 0.40 & 0.30 & 0.10 \\
$\phi_{\text {Model }}-\phi_{\text {Vis }}$ & -0.17 & -0.15 & +0.14 & -0.17 \\
Best $R_{\text {in }} / R_{\text {Phot }}$ & 1.8 & 2.0 & 2.2 & 2.4 \\
Best $\tau_{V}\left(\mathrm{Al}_{2} \mathrm{O}_{3}\right)$ & 2.5 & 2.5 & 1.5 & 1.5 \\
Best $\tau_{V}(\mathrm{Silicate})$ & 0 & 0 & 0 & 0 \\
Best $p$ & 3.5 & 3.5 & 3.0 & 2.5 \\
$\Theta_{\text {Phot }}[\mathrm{mas}]$ & 9.00 & 7.92 & 9.66 & 9.48 \\
$\chi_{v}^{2}$ & 0.16 & 0.10 & 0.43 & 0.35 \\
$n$ & 62 & 186 & 124 & 62 \\
$\sigma_{\text {formal }}[\mathrm{mas}]$ & 0.10 & 0.05 & 0.02 & 0.07 \\
$\sigma_{\text {model }}[\mathrm{mas}]$ & 0.30 & 0.09 & 0.06 & 0.34 \\
\hline & & & &
\end{tabular}

and that the exact choice of the outermost model radius is not critical for our calculation. Visibility $\left(V_{c}^{\text {dust }}\right)$ and flux $\left(f_{c}^{\text {dust }}\right)$ values for each spectral channel of MIDI were computed in the same way as for the dust-free model atmosphere, but without the extinction term $\mathrm{e}^{-\tau(v)}$.

Comparison of visibility and flux values to our data Finally, the flux and visibility values of the overall model were computed as

$f_{c}^{\text {total }}=f_{c}^{\text {star }}+f_{c}^{\text {dust }}$

and

$V_{c}^{\text {total }}=\frac{f_{c}^{\text {star }}}{f_{c}^{\text {total }}} V_{c}^{\text {star }}+\frac{f_{c}^{\text {dust }}}{f_{c}^{\text {total }}} V_{c}^{\text {dust }}$.

This simple addition of the CLVs of the dust-free model atmosphere of the $\mathrm{M}$ series and of the pure dust-shell (i.e. after subtraction of the contribution by the attenuated input radiation) is fully valid as long as the stellar atmosphere and dust shell are spatially separated. In fact, there may be some overlap of the extended low-intensity atmospheric molecular layers of the dustfree atmosphere model and the dust shell in our overall models. However, we verified that the inner boundary radius of the dust shell is clearly larger than that of the layer at which the atmosphere becomes optically thick at mid-infrared wavelengths. Ireland \& Scholz (2006) find for similar conditions that the gas temperatures for a dust-free $\mathrm{M}$ model and an $\mathrm{M}$ model that includes re-heating of gas by dust, differ by less than $50 \mathrm{~K}$ for radii up to about 3 parent-star radii.

For any given overall model separately, we optimized the angular diameter $\Theta_{\text {Phot }}$ using a standard least-square fit that minimizes the $\chi^{2}$ value between synthetic and observed visibility and flux values. Hereby, $\Theta_{\text {Phot }}$ was treated as the only free parameter. The resulting reduced $\chi_{v}^{2}$ values among our full grid of different overall models were compared in order to find the best-fitting set of model parameters. This analysis was separately performed for each of our epochs A-D.

\subsection{MIDI model results}

Table 3 lists for each of our MIDI epochs the parameter set of the best-fitting overall model of our grid. Listed are for each of our 4 epochs the best-fitting atmosphere model of the M series; its model phase; the difference between observed and model phase; the best-fitting parameters of the dust shell $\left(\tau_{V}\left(\mathrm{Al}_{2} \mathrm{O}_{3}\right), \tau_{V}(\right.$ Silicate $), \tau_{V}($ Silicate $\left.), p\right)$; the best-fitting angular diameter $\Theta_{\text {Phot }}$; the reduced $\chi_{v}^{2}$ value; the number of data points used $(n)$; and the formal and model errors. The model errors are
Table 4. Reduced $\chi_{v}^{2}$ values obtained for our grid of overall models.

\begin{tabular}{|c|c|c|c|c|c|}
\hline & & $\overline{\mathrm{A}}$ & $\overline{\mathrm{B}}$ & $\bar{C}$ & $\overline{\bar{D}}$ \\
\hline \multirow{6}{*}{ M model } & M20 & 0.27 & 0.16 & 0.52 & 0.38 \\
\hline & M21n & 0.26 & 0.14 & 0.53 & 0.35 \\
\hline & M22 & 0.16 & 0.17 & 0.81 & 0.51 \\
\hline & M23n & 0.17 & 0.12 & 0.43 & 0.61 \\
\hline & M24n & 0.20 & 0.10 & 0.66 & 1.04 \\
\hline & M25n & 0.19 & 0.17 & 0.53 & 0.58 \\
\hline \multirow[t]{5}{*}{$R_{\text {in }} / R_{\text {Phot }}$} & 1.8 & 0.16 & 0.10 & 0.56 & 0.54 \\
\hline & 2.0 & 0.19 & 0.10 & 0.52 & 0.46 \\
\hline & 2.2 & 0.21 & 0.11 & 0.43 & 0.39 \\
\hline & 2.4 & 0.24 & 0.12 & 0.50 & 0.35 \\
\hline & 2.6 & 0.29 & 0.18 & 0.57 & 0.38 \\
\hline \multirow[t]{5}{*}{$\tau_{V}\left(\mathrm{Al}_{2} \mathrm{O}_{3}\right)$} & 1.0 & 1 & 1 & 0.77 & 0.48 \\
\hline & 1.5 & 0.18 & 0.11 & \begin{tabular}{|l|}
0.43 \\
\end{tabular} & 0.35 \\
\hline & 2.0 & 0.17 & 0.11 & 0.56 & 0.54 \\
\hline & 2.5 & 0.16 & 0.10 & 0.80 & 0.71 \\
\hline & 3.0 & 0.18 & 0.11 & I & I \\
\hline \multirow{2}{*}{$\begin{array}{l}\tau_{V}(\text { Silicate }) / \\
\tau_{V}\left(\mathrm{Al}_{2} \mathrm{O}_{3}\right) \\
\end{array}$} & $0 \%$ & 0.16 & 0.10 & 0.43 & 0.35 \\
\hline & $10 \%$ & 0.19 & 0.12 & 0.52 & 0.38 \\
\hline \multirow[t]{5}{*}{$p$} & 2.0 & 0.31 & 0.20 & 0.87 & 0.38 \\
\hline & 2.5 & 0.20 & 0.14 & 0.54 & 0.35 \\
\hline & 3.0 & 0.17 & 0.10 & \begin{tabular}{|l|}
0.43 \\
\end{tabular} & 0.39 \\
\hline & 3.5 & 0.16 & 0.10 & 0.50 & 0.51 \\
\hline & 4.0 & 0.20 & 0.12 & I & I \\
\hline
\end{tabular}

derived as the standard deviations of $\Theta_{\text {Phot }}$ values based on different overall models of our grid that are consistent at the $1 \sigma$ level based on application of the $F$-test. The model errors of epochs A and $\mathrm{D}$ are larger than those of epochs $\mathrm{B}$ and $\mathrm{C}$, which can be explained by the use of only one baseline configuration at these epochs. Table 4 lists for each epoch and for each grid parameter the best $\chi_{v}^{2}$ value that could be obtained by any combination of all other parameters. The best $\chi_{v}^{2}$ value for each epoch is marked by boxes.

The synthetic visibility and flux values corresponding to the best-fitting overall models are shown in Figs. 2-5 in comparison to the measured values. Panels (d), which show the equivalent UD diameter values, also indicate the photospheric angular diameter $\Theta_{\text {Phot }}$ and the inner dust shell boundary $\Theta_{\text {in }}$ derived from the model fit. Panels (e) show the intensity profiles of the overall models that fit our MIDI data best for three bandpasses at $8-9 \mu \mathrm{m}, 10-11 \mu \mathrm{m}$, and $12-13 \mu \mathrm{m}$. These panels have a bottom and a top abscissa indicating (bottom) the radius as a function of the model photospheric radius $R_{\text {Phot }}$ and (top) the corresponding angular scale using the best-fitting angular diameter $\Theta_{\text {Phot }}$. Panels (e) also indicate the inner dust shell radius $R_{\text {in }}$, as well as the mean $\mathrm{SiO}$ maser ring radii $R_{42.8}$ and $R_{43.1}$, which are derived in Sect. 4. A two-dimensional pseudo-color image of the mid-infrared model intensity showing the molecular layers and the dust shell is displayed in Fig. 7. The photospheric disk is represented by a light blue color. Overlaid are the images of the $\mathrm{SiO}$ maser radiation described in Sect. 4.

The photospheric diameter $\Theta_{\mathrm{Phot}}$ The obtained photospheric diameter values $\Theta_{\mathrm{Phot}}$ at phases $0.42,0.55,1.16$, and 1.27 are $9.0 \mathrm{mas} \pm 0.3 \mathrm{mas}, 7.9 \mathrm{mas} \pm 0.1 \mathrm{mas}, 9.6 \mathrm{mas} \pm 0.1 \mathrm{mas}$, and 9.5 mas \pm 0.4 mas, respectively. Here, the error bars include the formal, as well as the model, errors from Table 3. Our measurements indicate significant changes of the photospheric diameter $\Theta_{\text {Phot }}$ as a function of stellar phase. A detailed comparison 
of $\Theta_{\text {Phot }}$ to the inner dust shell radius and the $\mathrm{SiO}$ maser ring radii follows in Sect. 5 .

In BW05, we estimated S Ori's photospheric angular diameter at phase 0.73 to $\sim 9.2 \mathrm{mas}$, based on our $K$-band UD diameter measurements and using correction factors from UD to photospheric diameters from Ireland et al. (2004a,b). This value is consistent with the photospheric diameters derived here, as the estimate of 9.2 mas at phase 0.73 from BW05 lies as expected in between the values of 7.9 mas at phase 0.54 and 9.6 mas at phase 1.16 derived in the present paper.

The $K$-band UD diameter of 10.54 mas \pm 0.68 mas by van Belle et al. (1996) was obtained at phase 0.56. At phases close to this value, the $\mathrm{M}$ series results in a ratio between the $K$-band UD diameter and the photospheric diameter of $\sim 1.35$ (Fig. 7 of Ireland et al. 2004b). With this correction, the photospheric diameter corresponding to this measurement at phase 0.56 would be 7.8 mas \pm 0.5 mas, and would thus be fully consistent with our value of 7.9 mas \pm 0.1 mas at the same phase (Epoch B at phase 0.54).

Millan-Gabet et al. (2005) obtained a $K$-band UD diameter of $9.6 \mathrm{mas} \pm 0.2 \mathrm{mas}$ at phase 0.1 . At this phase, the ratio between $K$-band UD diameter and photospheric diameter is predicted to be close to unity (Ireland et al. 2004b). This measurement thus also agrees well with our photospheric diameter of 9.6 mas \pm 0.4 mas at phase 1.16 (epoch C).

The good agreement of the photospheric angular diameters derived from our MIDI measurements in the present paper with previous near-infrared diameter measurements increases confidence in the validity of our modeling using the $\mathrm{M}$ model series complemented by a radiative transfer model of the dust shell.

The choice of the $M$ model The intensity profile of the extended atmosphere, and thereby the choice of the best-fitting model out of the $\mathrm{M}$ series, is mainly constrained by the shape of the visibility functions, in particular for longer baseline lengths, in the wavelength range $\sim 8-9 \mu \mathrm{m}$, where the contribution by the dust shell is relatively low.

The differences in visual stellar phase of the best-fitting M model compared to the visual phase at the epochs of observation are $-0.17,-0.15,+0.14$, and -0.17 , respectively. These differences may be due to the uncertainties of the absolute assignments of visual phases to the model series as well as to the dates of observation, which are both on the order of 0.1. The relative phase values are expected to agree better, which is true for epochs A, B, and D. Differences between these phases might also occur owing to the different radius and pulsation period of the model series' parent star compared to S Ori. These parameters might have an effect on the extension of the atmosphere, so that the best-fitting extension of the atmosphere might belong to a model at a different visual phase. An effect such as this might explain the clearly different phase difference between observation and model at epoch C compared to the three other epochs.

The good agreement of the shape of the synthetic visibility function with our observations at the wavelength range from $8 \mu \mathrm{m}$ to $9 \mu \mathrm{m}$ and the simultaneous agreement with the measured flux level again confirms that the $\mathrm{M}$ model series is usable for S Ori.

As an indication of the extension of the molecular layers of the best-fitting $\mathrm{M}$ models, the $50 \% / 10 \%$ intensity radii of the $N$-band $(8-13 \mu \mathrm{m})$ model CLVs are 1.5/1.6, 1.8/1.9, 1.6/1,7, and 1.4/1.9 photospheric radii $R_{\text {Phot }}$ for epochs A-D, i.e. for models M22, M24n, M23n, and M21n, respectively.
The dust shell chemistry The ratio of the number of $\mathrm{Al}_{2} \mathrm{O}_{3}$ grains to that of silicate grains is mostly constrained by the shape of the flux and visibility spectra at the silicate feature close to $10 \mu \mathrm{m}$. We obtain best fits to our S Ori data with a dust shell only consisting of $\mathrm{Al}_{2} \mathrm{O}_{3}$ grains. The addition of small amounts of silicate grains does not improve the fits for any of our epochs. While our grid in Table 4 only includes silicate grains with the same inner boundary radius as for the $\mathrm{Al}_{2} \mathrm{O}_{3}$ grains, we also compared to our data the best-fitting models from Table 3 with silicate grains added at larger inner boundary radii of 4 and 6 photospheric radii. These models lead to clearly increased $\chi_{v}^{2}$ values as well. In summary, our data indicate that silicon is not bound in dust, neither at very close distances of $\sim 2 R_{\text {Phot }}$ nor at larger distances of $4-6 R_{\text {Phot }}$. A dust shell only consisting of $\mathrm{Al}_{2} \mathrm{O}_{3}$ grains is consistent with the modeling of the IRAS LRS spectra of S Ori by Lorenz-Martins \& Pompeia (2000).

The radial density profile of the dust shell The total optical depth of the dust shell is mainly constrained by the height and shape of the MIDI total flux spectrum. The radial density profile described by the inner boundary radius and the density gradient is mostly constrained by the shape of the visibility function versus wavelength. Here, the values at shorter wavelengths, probing warmer dust, constrain the inner boundary radius best. The density gradient is mostly constrained by the visibility values at longer wavelengths and shorter baseline lengths, with which the dust shell is less resolved.

We obtain best-fitting inner boundary radii of 1.8, 2.0, 2.2, and 2.4 photospheric radii $R_{\text {Phot }}$ at our phases $0.42,0.55,1.16$, and 1.27, respectively. These radii are well-constrained and do not significantly change for variations in other model parameters. We estimate the uncertainty of this parameter to about $0.2 \times$ $R_{\text {Phot }}$. The formation of dust consisting of $\mathrm{Al}_{2} \mathrm{O}_{3}$ grains at these short distances from the stellar surface is consistent with the empirical results by Lorenz-Martins \& Pompeia (2000), as well as with the recent theoretical calculations by Ireland \& Scholz (2006) and Woitke (2006).

Best-fitting density gradients $p$ at the same phases are 3.5, $3.5,3.0$, and 2.5, respectively, and best-fitting optical depths $\tau_{V}$ are $2.5,2.5,1.5$, and 1.5 . Taking the inner boundary radii, the density gradients, and the optical depth values together, our fits indicate more compact dust shells with larger optical depth near the stellar minimum and more extended dust shells with lower optical depth after stellar maximum.

Wind models We used the radiation transport code DUSTY (Ivezić \& Elitzur 1997; Ivezić et al. 1999) with its option of modeling radiatively driven winds to compute the density structure of S Ori's envelope that would result from a wind model. We computed wind models for each of our epochs A to D using a blackbody with effective temperature of the best-fitting M model as the central radiation source and with the $R_{\text {in }}$ and $\tau_{V}$ values from Table 3 . We used the same optical properties of $\mathrm{Al}_{2} \mathrm{O}_{3}$ as above, and we set $R_{\text {out }} / R_{\text {in }}$ to $10^{3}$. Figure 6 shows the resulting normalized density profiles of the wind models together with the $\rho \propto r^{-2}$ and $\rho \propto r^{-3}$ curves. The wind models result in density structures $\rho \propto r^{-2}$ for $r \gtrsim 10 R_{\text {Phot }}$. Closer to the photosphere, the gradients of the wind density profiles become steeper than $\rho \propto r^{-3}$. As our MIDI visibility measurements are mostly sensitive to the inner dust region, the relatively high density gradients given in Table 3 could be explained by such a wind model. The differences among the empirically found density gradients for the different epochs could be explained by 


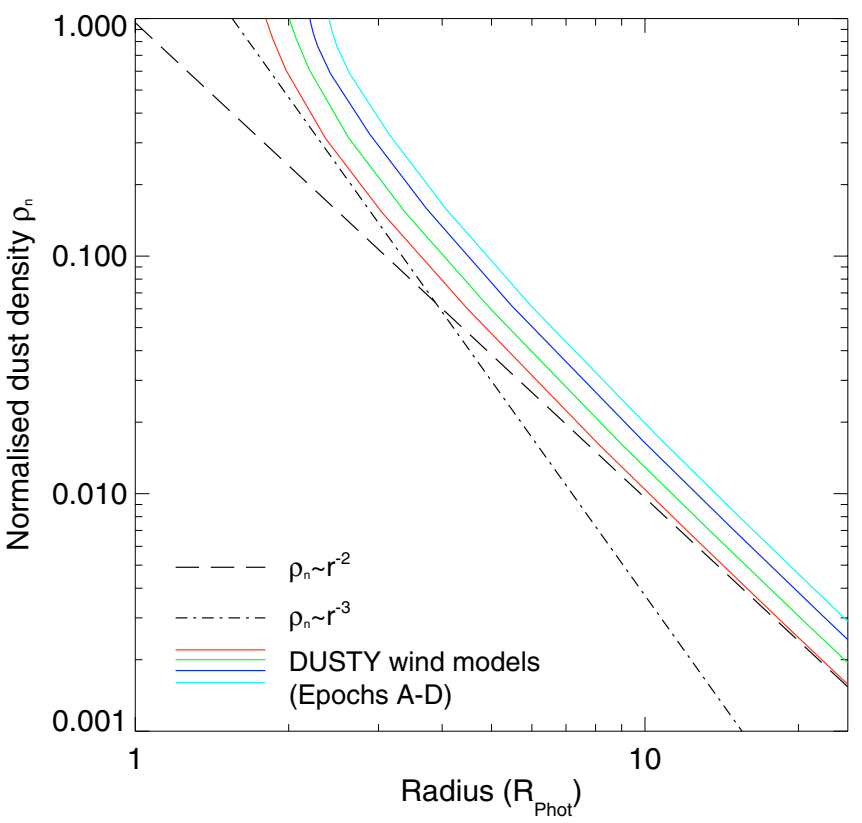

Fig. 6. Density profiles of radiatively driven wind models calculated with the DUSTY code (Ivezić \& Elitzur 1997; Ivezić et al. 1999) for input parameters from Table 3, together with $\rho \propto r^{-2}$ and $\rho \propto r^{-3}$ curves.

different mean distances from which most mid-infrared photons escape. The wind models shown correspond to mass-loss rates between $\sim 2.4 \times 10^{-6} M_{\odot} / \mathrm{yr}$ (Epoch C) and $\sim 3.5 \times 10^{-6} M_{\odot} / \mathrm{yr}$ (Epoch B). The indication of more compact dust shells with larger optical depth near stellar minimum and more extended dust shells with lower optical depth after stellar maximum thus suggests a wind with increased mass loss and dust formation occurring relatively close to the stellar surface near minimum visual phase and with an expanded dust shell after maximum phase.

Symmetry of the molecular and dust shells Our MIDI data at epochs $A$ and D are each obtained at one single position angle on sky. Epoch B spans a range in position angles of $\sim 40^{\circ}$ and epoch $\mathrm{C}$ of $\sim 20^{\circ}$. Within these probed ranges, our S Ori data can be described well by spherically symmetric distributions of molecular layers and dust shells without the need to introduce deviations from circular symmetry to our modeling. However, we cannot rule out asymmetries at wider ranges of position angles, in which case the resulting parameters in Table 3 are only valid along the position angles on the sky given in Table 1. Note that the distributions of the $\mathrm{SiO}$ maser spots toward S Ori shown in BW05, as well as below in Sect. 4, do not indicate a globally asymmetric gas distribution.

\section{VLBA measurements}

Contemporaneous with the VLTI/MIDI observations, we observed the $v=1, J=1-0(43.1 \mathrm{GHz})$ and $v=2, J=1-0(42.8 \mathrm{GHz}) \mathrm{SiO}$ maser emission associated with S Ori $\left(\alpha=05^{\mathrm{h}} 29^{\mathrm{m}} 00^{\mathrm{s}} .9, \delta=-04^{\circ} 41^{\prime} 32^{\prime \prime} \cdot 7, \mathrm{~J} 2000\right)$ using the 10 stations of the VLBA, which is operated by the National Radio Astronomy Observatory (NRAO). Table 5 lists the details of our $\mathrm{SiO}$ maser observations recorded over three epochs. Listed are the epoch, date, time, Mean Julian Date (MJD) of the start of observation, experiment duration, number of antennas, and the visual phase $\Phi_{\text {vis }}$. The first two
Table 5. VLBA observations of S Ori.

\begin{tabular}{llllrrr}
\hline \hline Ep. & Date & Time & MJD & $\begin{array}{r}\text { Dur. } \\
\text { (h) }\end{array}$ & $\begin{array}{r}\text { No. } \\
\text { Ant. }\end{array}$ & $\Phi_{\text {vis }}$ \\
\hline A & $2005-01-17$ & $02: 36$ & 53387.6 & 5.0 & 9 & 0.46 \\
B & $2005-02-28$ & $23: 47$ & 53430.5 & 2.6 & 10 & 0.56 \\
C & $2005-11-05$ & $07: 23$ & 53679.8 & 5.0 & 10 & 1.14 \\
\hline
\end{tabular}

epochs occurred near stellar minimum and were spaced 42 days apart or approximately $10 \%$ of the stellar period. The third epoch occurred roughly 9 months later just after maximum on the next stellar cycle. Data were recorded in a similar manner for all three epochs at reference frequencies of $43.122080 \mathrm{GHz}$ and $42.820587 \mathrm{GHz}$ for the $v=1$ and $v=2 \mathrm{SiO}$ transitions, respectively. Right- and left-hand circular polarization was used with $8-\mathrm{MHz}\left(56.1 \mathrm{~km} \mathrm{~s}^{-1}\right)$ bands centered on the local standard-of-rest (LSR) velocity of $18.0 \mathrm{~km} \mathrm{~s}^{-1}$. Interspersed with observations of S Ori were 10-min scans of extragalactic continuum sources $(0423-013$ and $0359+509)$ for the purpose of delay and bandpass calibration. For epoch A only 9 of the 10 stations of the VLBA were available for our observations. Unfortunately, the one antenna that was unavailable was the station at Mauna Kea, HI. This station when combined with the St. Croix, VI antenna provides the longest baseline (greatest resolution) in the array. For this reason the resolution of the epoch A images is reduced from that of the other two epochs. There was also a problem with the second epoch (epoch B) of observations in that roughly half of the data were accidentally lost after the correlation. Despite this loss, we were still able to use the remaining data to produce quality images of the masers. NRAO kindly scheduled a third epoch of observations (epoch C) to make up for the loss.

\subsection{VLBA data reduction and analysis}

The data were correlated at the VLBA correlator operated by NRAO in Socorro, New Mexico. Auto and cross-correlation spectra consisting of 256 channels with channel spacings of $31.25 \mathrm{kHz}\left(\sim 0.2 \mathrm{~km} \mathrm{~s}^{-1}\right)$ were produced by the correlator. Calibration was performed using the Astronomical Image Processing System (AIPS) maintained by NRAO. The data were calibrated in a manner similar to what was performed in BW05. For each epoch, residual delays due to the instrumentation were corrected by performing a fringe fit on the continuum calibrator scans. Residual group delays for each antenna were determined and applied to the spectral line data. Variations in the residual delays ranged from $2-4 \mathrm{~ns}$ resulting in phase errors of no more than $1.5-3^{\circ}$ across the $8-\mathrm{MHz}$ band.

The bandpass response was determined from scans on the continuum calibrators and used to correct the target-source data. The time-dependent gains of all antennas relative to a reference antenna were determined by fitting a total-power spectrum (from the reference antenna with the target source at a high elevation) to the total power spectrum of each antenna. The absolute flux density scale was established by scaling these gains by the system temperature and gain of the reference antenna. Errors in the gain and pointing of the reference antenna and atmospheric opacity variations contribute to the error in the absolute amplitude calibration, which is accurate to about $15-20 \%$.

Residual fringe rates were obtained by fringe-fitting a strong reference feature in the spectrum of each maser transition. For epoch $\mathrm{A}$ we used the channel at velocity $V_{\mathrm{LSR}}=$ $12.3 \mathrm{~km} \mathrm{~s}^{-1}$ for the $v=1$ transition and the channel at velocity $V_{\mathrm{LSR}}=16.9 \mathrm{~km} \mathrm{~s}^{-1}$ for the $v=2$ transition. For epoch B we used channels at $V_{\mathrm{LSR}}=12.8 \mathrm{~km} \mathrm{~s}^{-1}$ and $V_{\mathrm{LSR}}=12.1 \mathrm{~km} \mathrm{~s}^{-1}$ 

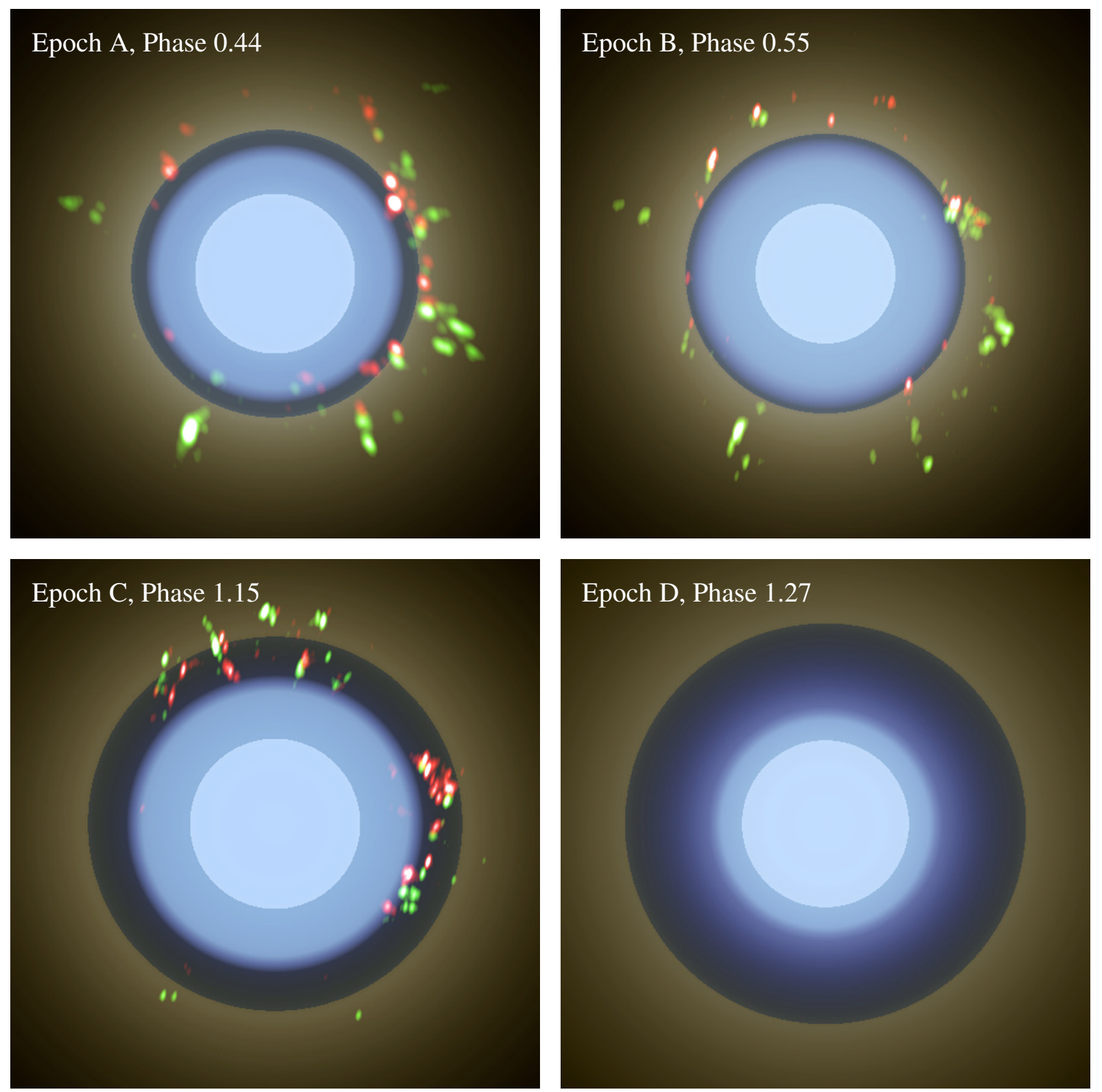

Fig. 7. The (red) $v=2, J=1-0(42.8 \mathrm{GHz})$ and (green) $v=1, J=1-0(43.1 \mathrm{GHz})$ maser images overlaid onto pseudo-color representations of the infrared intensity. The continuum photosphere (in fact mostly hidden behind the molecular atmosphere in the $N$-band) is enhanced to a light blue color. The darker blue shades represent our model intensity profile as in panels (e) of Figs. 2-5, and the green shades represent the location of the $\mathrm{Al}_{2} \mathrm{O}_{3}$ dust shell on top of the low-intensity extended wings of the molecular atmosphere. The true location of the star relative to the maser images is unknown. Here, we assume that the center of the star coincides with the center of the maser spot distribution. Synthesized beam sizes for epoch A are $0.59 \times 0.40$ mas and $0.60 \times 0.37$ mas for the $v=1$ and $v=2$ transitions, respectively. For epoch B these sizes are $0.52 \times 0.21$ mas and $0.51 \times 0.20$ mas, and for epoch C $0.46 \times 0.16$ mas and $0.44 \times 0.16$ mas, respectively. Epoch D is a MIDI-only epoch and SiO maser observations have not been obtained at this epoch. The size of each panel is $30 \times 30$ mas, corresponding to $14.4 \times 14.4 \mathrm{AU}$.

for the $v=1$ and $v=2$ transitions, respectively. Finally, for epoch $\mathrm{C}$ we used the same velocity channel for both transitions $V_{\mathrm{LSR}}=10.3 \mathrm{~km} \mathrm{~s}^{-1}$. The resulting fringe-rate solutions were applied to all channels in each spectrum. An iterative selfcalibration and imaging procedure was then performed to map this reference channel for each transition. The resulting residual phase and amplitude corrections from the reference channels at $42.8 \mathrm{GHz}$ and $43.1 \mathrm{GHz}$ were applied to all channels in the respective bands. This procedure was repeated for each transition at each epoch.

In order to accurately compare the distributions of the two maser transitions, it is desirable to determine a common spatial reference point. However, after the fringe-fitting step to determine residual fringe-rates, all absolute position information is lost for the VLBA data. To accomplish this registration, we used the same technique employed in BW05 with the residual fringe-rates, phases, and amplitudes from one transition (e.g., $v=2,42.8 \mathrm{GHz}$ ) used to calibrate the other transition (e.g., $v=1,43.1 \mathrm{GHz})$ in order to determine an offset between the two phase centers.

The final image cubes of the $\mathrm{SiO}$ maser emission consisting of $1024 \times 1024$ pixels $(\sim 51 \times 51$ mas $)$ were generated for each transition at each of the three epochs. Images were produced for all spectral channels containing $\mathrm{SiO}$ maser emission in each transition at each epoch. Synthesized beam sizes used for epoch A were $0.59 \times 0.40$ mas and $0.60 \times 0.37$ mas for the $v=1$ 
and $v=2$ transitions, respectively. For epoch B these sizes were $0.52 \times 0.21$ mas and $0.51 \times 0.20$ mas. For epoch $\mathrm{C}$ the beam sizes used in the imaging were $0.46 \times 0.16$ mas and $0.44 \times 0.16$ mas. Note that the resolution was greatly reduced in epoch A due to the loss of the Mauna Kea, HI VLBA antenna mentioned previously. The resolution of epoch B is slightly worse than that of epoch $\mathrm{C}$ due to the loss of half the data, thus reducing the $u v$ coverage of the array. Using the procedure described above, the image cubes from the two transitions were spatially aligned for each epoch. Off-source rms noise estimates in the images ranged from $5 \mathrm{mJy}$ to $9 \mathrm{mJy}$ for epoch A, $3 \mathrm{mJy}$ to $7 \mathrm{mJy}$ for epoch B, and $7 \mathrm{mJy}$ to $18 \mathrm{mJy}$ for epoch $\mathrm{C}$. Figure 7 shows the total intensity images of the $v=1,43.1-\mathrm{GHz}$ and $v=2,42.8-\mathrm{GHz}$ $\mathrm{SiO}$ maser emission toward $\mathrm{S}$ Ori overlaid onto a pseudo-color representation of the mid-infrared intensity profile obtained in Sect. 3.

The six resulting image cubes ( 2 transitions at 3 epochs) were then analyzed to extract relevant maser parameters. Twodimensional Gaussian functions were fit to maser emission above a cut-off flux density of $100 \mathrm{mJy}$ in each spectral (velocity) channel using the AIPS task SAD. This fitting yielded velocities, flux densities, and positions in right ascension and declination for all emission components identified in the images. Errors in right ascension and declination of identified components were computed using the fitted source size divided by twice the signalto-noise ratio $(\mathrm{s} / \mathrm{n})$ in the image and ranged from $1 \mu$ as for features with high $\mathrm{s} / \mathrm{n}$, to $28 \mu$ as for features with lower $\mathrm{s} / \mathrm{n}$.

The remaining analysis of the maser component identifications was performed outside of the AIPS package. For comparison with the total flux densities derived from the MIDI data, we summed the flux densities and their errors for all fitted components in all channels for each transition/epoch. These integrated flux densities are discussed further in Sect. 5. Since the $\sim 0.2 \mathrm{~km} \mathrm{~s}^{-1}$ channel spacing is sufficient to resolve the masers spectrally, features typically appear in multiple adjacent spectral channels. Positions in right ascension and declination and center velocities for the masers were determined using a flux-densitysquared weighted average for features identified in two or more adjacent channels with a spatial coincidence of 0.5 mas. The flux assigned to the maser averages was the maximum single-channel flux density. The results from the image analysis and component averaging are described in the next section.

\subsection{VLBA results}

\subsubsection{The spatial structure of the $\mathrm{SiO}$ masers}

The maser features characterized using the procedures described above are listed in Tables A.2-A.4 and are shown in Fig. 8, where there are six panels ordered by epoch left to right and by transition top to bottom. Within each panel there are two subpanels. Each upper sub-panel shows the spectrum of the maser emission with flux density plotted as a function of local standard of rest (LSR) velocity. Point sizes are proportional to the logarithm of the flux density and are color-coded according to velocity bin in increments of $1.7 \mathrm{~km} \mathrm{~s}^{-1}$. The bottom sub-panels of Fig. 8 show the spatial distribution of the $\mathrm{SiO}$ masers as observed in the images. Point sizes are again proportional to the logarithm of the flux density with the same velocity color-coding as in the top sub-panels.

Examining the upper sub-panels and the component tables, we find that the masers have shifted slightly toward the blue end of the spectrum from our previous BW05 observations. There the maser velocities ranged from 11.1 to $24.7 \mathrm{~km} \mathrm{~s}^{-1}$, while for these latest three epochs the masers span a velocity range from 8.6 to $19.0 \mathrm{~km} \mathrm{~s}^{-1}$. The velocity range for our latest three epochs is consistent with earlier spectral measurements by Jewell et al. (1991), who measured a velocity range of 7.1 to $19.6 \mathrm{~km} \mathrm{~s}^{-1}$ for the $v=1$ masers. Spectral ranges measured by Cho et al. (1996) were much narrower at $11.0-16.0 \mathrm{~km} \mathrm{~s}^{-1}$ for the $v=1$ and $12.0-14.7 \mathrm{~km} \mathrm{~s}^{-1}$ for the $v=2$ masers, respectively. For the three epochs discussed here, flux densities of the peak component in the spectrum ranged from 3.0 to $5.4 \mathrm{Jy} \mathrm{beam}^{-1}$ for the $v=1$ masers and 1.3 to $6.4 \mathrm{Jy} \mathrm{beam}^{-1}$ for the $v=2$ masers. These are close to our BW05 results for which we measured peaks of $4.3 \mathrm{Jy}$ beam $^{-1}$ and $5.3 \mathrm{Jy}_{\text {beam }}{ }^{-1}$ for the $v=1$ and $v=2$ masers, respectively.

When compared to the spectrally-averaged total-intensity images (Fig. 7), the maser component maps in lower sub-panels of Fig. 8 provide an accurate representation of the spatial structure of the maser emission summed over all velocity channels in the image cube. The component maps all show that the $\mathrm{SiO}$ masers in both transitions form partial to full rings of emission with the typical clumpy distribution. The top three panels show the $v=1, J=1-0$ to form a partial ring for epoch A with a gap to the north, nearly a full ring for epoch $\mathrm{B}$, and again a partial ring dominated by emission in the northern and western regions for epoch $\mathrm{C}$. For the $v=2, J=1-0 \mathrm{SiO}$ masers shown in the bottom three panels, we observe nearly a full ring of emission for epoch A, a partial ring with a gap to the south for epoch B, and a partial ring dominated by northern and western features for epoch $\mathrm{C}$. In all three epochs the $v=1$ masers appear to be distributed over a larger area on the sky than the $v=2$ masers. In addition, the $v=1$ rings appear thicker than the corresponding $v=2$ rings at each epoch. It should be noted that, while epochs A and B were spaced only 42 days apart, there are significant differences in the number and distribution of the masers in both transitions. This is not unexpected since previous multi-epoch VLBI studies (e.g. Diamond \& Kemball 2003) have shown the fine structure of the $\mathrm{SiO}$ masers to vary significantly over periods as short as two weeks. This variation, combined with the relative complexity of the maser structures, precluded us from tracking individual maser components in order to determine maser proper motions.

To better characterize the apparent size and thickness of the $\mathrm{SiO}$ maser distributions, we computed the average angular distance of the masers from the center of their distribution $(\bar{r})$. To accomplish this, we first determined the center of the distribution by performing a least-square fit of a circle to the combined $v=1$ and $v=2$ maser positions. We were able to combine the two sets of masers only because we had previously aligned the images of the two transitions following the procedure described in Sect. 4.1. This fit produced a common center from which we computed the mean maser angular distance $\bar{r}_{\mathrm{SiO}}$ and the standard deviation for each transition independently for each epoch.

The mean angular distances from the center for the observed $\mathrm{SiO}$ masers at $v=1,43.1 \mathrm{GHz}$ and $v=2,42.8 \mathrm{GHz}$ are listed in Table 6 . These distances are also indicated by a dashed circle in each of the six panels in Fig. 8. The standard deviations in the mean distances are also listed in Table 6 and represented in Fig. 8 as the error on the mean. Two times the standard deviation also provides an indication of the thickness of the shell. The listed parameters in Table 6 are for each epoch of observation/visual phase and each of the two transitions, the apparent mean angular distances from the center of the masers spots $\bar{r}_{v=1,2}$, the ratio of the mean distances of the two transitions $\bar{r}_{v=1} / \bar{r}_{v=2}$, as well as the mean LOS velocities $\bar{V}_{v=1,2}$ and their ratio $\bar{V}_{v=1} / \bar{V}_{v=2}$. 

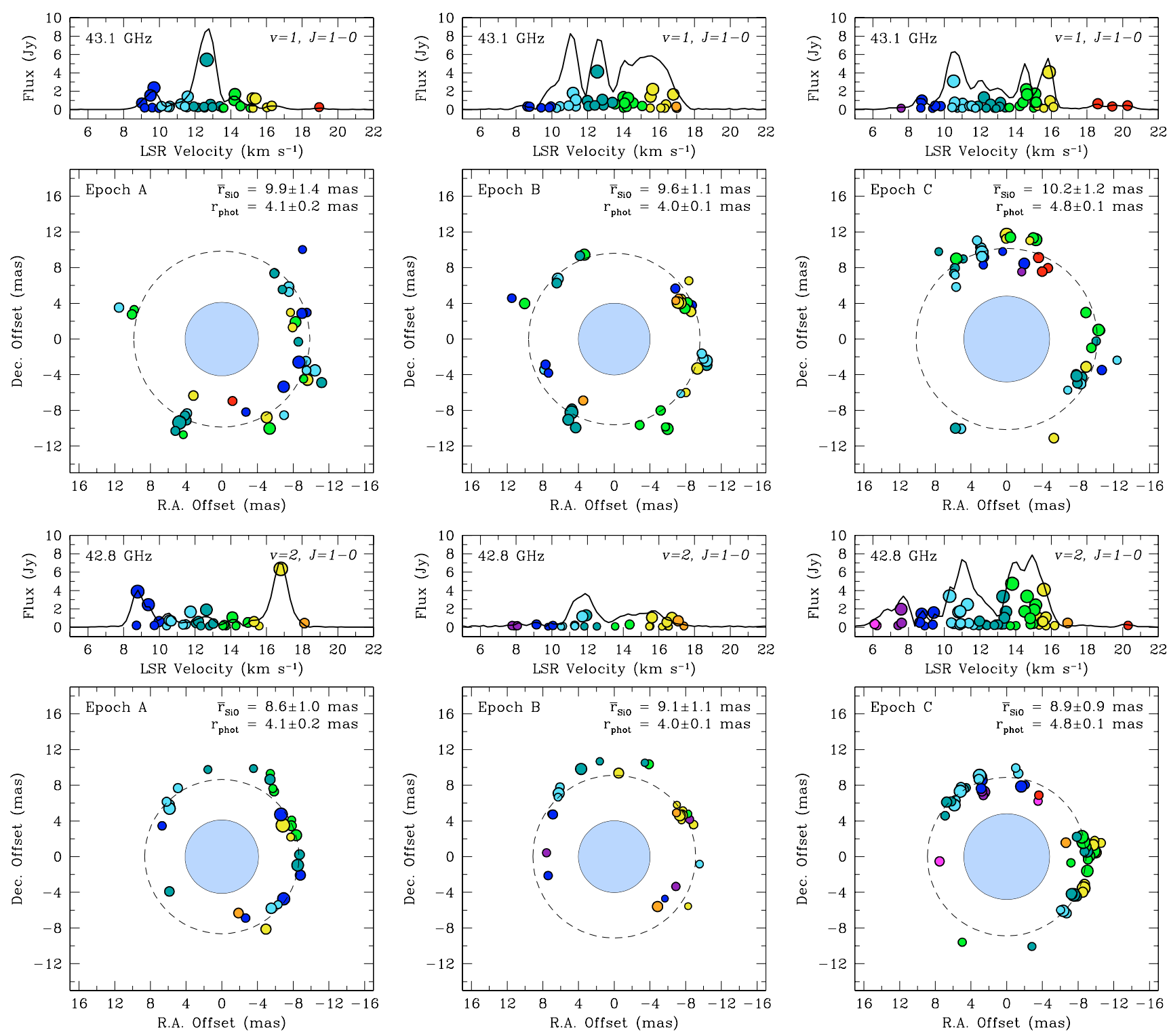

Fig. 8. LOS velocity structure of the $(t o p) v=1, J=1-0(43.1 \mathrm{GHz})$ and (bottom) $v=2, J=1-0(42.8 \mathrm{GHz}) \mathrm{SiO}$ maser emission toward S Ori. The epochs of observations are from left to right Epoch A $\left(\Phi_{\text {vis }}=0.46\right)$, Epoch B $\left(\Phi_{\text {vis }}=0.56\right)$, and Epoch C $\left(\Phi_{\text {vis }}=1.14\right)$. The top sub-panels of each epoch and transition show the spectra formed by plotting maser intensity versus velocity, color-coded in $1.7 \mathrm{~km} \mathrm{~s}{ }^{-1} \mathrm{velocity}$ increments from redward to blueward. The solid lines in the top sub-panels represent the scalar-averaged cross-power spectra averaged over all of the VLBA antennas. The bottom (main) sub-panels plot the spatial and velocity distributions of the masers. The color of each point represents the corresponding velocity bin in the spectrum, and the size of each point is proportional to the logarithm of the flux density. Errors in the positions of the features are smaller than the data points. The dashed circles are based on the mean angular distances of the SiO masers from the centers of the distributions. The light blue filled circles in the centers illustrate for comparison the angular sizes of the continuum photosphere derived in Sect. 3.4. The true location of the star relative to the masers is unknown and here assumed to coincide with the center of the maser spot distribution. We use also panels (e) of Figs. 2-5, as well as Fig. 7, for a comparison of the SiO maser ring radii to the extension of the molecular atmosphere and the inner dust shell boundary.

From Table 6 we see that $\bar{r}$ ranges from 8.6 to 10.2 mas. At every epoch we find that the $v=1$ masers have a greater mean distance from center than the $v=2$ masers. This finding is consistent with BW05 in which we found $\bar{r}_{v=1}=9.4$ mas and $\bar{r}_{v=2}=8.8$ mas. In BW05, however, we were unable to make a definitive statement about the relative sizes of the two maser distributions because we had only the one epoch of observations, and at this epoch, the $v=2$ masers were primarily confined to a small region on the northwest side of the shell. In our more recent epochs, the rings of emission are much more complete making the comparison between transitions easier. Although the differences in $\bar{r}$ for epochs A, B, and C are less than the standard deviation (thickness of the ring), they are still greater than the resolution of the images given earlier by the synthesized beam sizes of $0.6 \times 0.4$ mas for epoch A and $0.5 \times 0.2$ mas for epochs B and $\mathrm{C}$. The fact that the $v=1$ ring is consistently larger than the $v=2$ ring suggests that for S Ori the two sets of masers are not co-spatial.

The relative separation of the $v=1$ and $v=2$ masers for S Ori is also consistent with recent results obtained for the $\mathrm{SiO}$ masers around other stars including: IRC +10011 (Desmurs et al. 2000); TX Cam (Desmurs et al. 2000; Yi et al. 2005); $o$ Cet, U Ori, and R Aqr (Cotton et al. 2006). In addition, numerical simulations of $\mathrm{SiO}$ masers (Humphreys et al. 1996; 
Table 6. Results from our VLBA observations of the $v=1,43.1 \mathrm{GHz}$ and $v=2,42.8 \mathrm{GHz} \mathrm{SiO}$ maser transition toward $\mathrm{S}$ Ori.

\begin{tabular}{lrrr}
\hline \hline Epoch & $\mathrm{A}$ & $\mathrm{B}$ & $\mathrm{C}$ \\
Phase $\Phi_{\text {vis }}$ & 0.46 & 0.56 & 1.14 \\
\hline $\bar{r}_{v=1}[\mathrm{mas}]$ & $9.9 \pm 1.4$ & $9.6 \pm 1.1$ & $10.2 \pm 1.2$ \\
$\bar{r}_{v=2}[\mathrm{mas}]$ & $8.6 \pm 1.0$ & $9.1 \pm 1.1$ & $8.9 \pm 0.9$ \\
$\bar{r}_{v=1} / \bar{r}_{v=2}$ & 1.15 & 1.05 & 1.15 \\
$\bar{V}_{v=1}\left[\mathrm{~km} \mathrm{~s}^{-1}\right]$ & $12.6 \pm 2.4$ & $13.3 \pm 2.5$ & $13.0 \pm 2.8$ \\
$\bar{V}_{v=2}\left[\mathrm{~km} \mathrm{~s}^{-1}\right]$ & $12.5 \pm 2.4$ & $12.9 \pm 3.2$ & $12.5 \pm 2.9$ \\
$\bar{V}_{v=1} / \bar{V}_{v=2}$ & 1.01 & 1.03 & 1.04 \\
\hline
\end{tabular}

Gray \& Humphreys 2000) show the $v=1, J=1-0$ masers to occur farther from the star than the $v=2, J=1-0$ masers throughout the entire pulsation. The simulations of Gray \& Humphreys (2000) also show that the mean thickness of the $v=1$ shell is roughly twice that of the $v=2$ shell throughout the stellar cycle. In our measurements, we find the $v=2$ shell to be thicker in the BW05 observations, the $v=1$ shell to be thicker in epochs $\mathrm{A}$ and $\mathrm{C}$, and the two transitions to be roughly equivalent in epoch B. In the two cases for which the $v=1$ shell was thicker, the ratio of the two thicknesses is 1.3-1.4, much less than the 2 determined by Gray \& Humphreys. The angular sizes of the maser shells are reported in Table 7. At the assumed distance for S Ori of $480 \mathrm{pc} \pm 120 \mathrm{pc}$ (van Belle et al. 2002), 1 mas is roughly equivalent to $0.48 \mathrm{AU}$. Thus the linear-scale sizes for maser distributions range from 8.3 to $9.8 \mathrm{AU}$ and the maser shell thickness (twice the standard deviation) ranges from roughly 0.9 to $1.3 \mathrm{AU}$.

\subsubsection{The kinematics of the SiO masers}

In BW05, we reported that the $\mathrm{SiO}$ masers showed no coherent velocity structure indicative of global expansion/infall or rotation. Examining Fig. 8, we again find no evidence of such coherence in the line-of-sight (LOS) velocities of the masers as a function of spatial location. We did, however, notice that there appears to be a velocity gradient at all epochs, with masers toward the blue- and red-shifted ends of the spectrum lying closer to the center of the distribution than masers at intermediate velocities. This phenomenon was also found in BW05 and has been previously observed in other $\mathrm{SiO}$ maser studies (e.g. Boboltz \& Marvel 2000; Hollis et al. 2001).

The LSR stellar velocity of S Ori has not been measured particularly well. In the General Catalog of Stellar Radial Velocities (Wilson 1953), the value listed for S Ori is $22 \mathrm{~km} \mathrm{~s}^{-1}$. Young (1995) observed the sub-millimeter CO (3-2) and $\mathrm{CO}$ (4-3) lines toward $\mathrm{S}$ Ori and determined values of $V_{\mathrm{LSR}}=$ $14.5 \pm 0.2$ and $14.1 \pm 0.5 \mathrm{~km} \mathrm{~s}^{-1}$ from fits to the spectra, respectively. Winters et al. (2003) derived $V_{\mathrm{LSR}}=14.0 \mathrm{~km} \mathrm{~s}^{-1}$ based on fits of the CO (1-0) and CO (2-1) lines. Since the $\mathrm{SiO}$ masers are not distributed evenly about any of the above velocities, we computed an average LOS velocity from the masers themselves for each transition at epoch. The values of $\bar{V}_{\mathrm{LOS}}$ for each of the epochs are reported in Table 6 with errors of $15 \%$ estimated from the uncertainty of the absolute amplitude calibration. Although the differences are well within the error bars, it appears that the $v=2$ masers are shifted slightly toward the blue end of the spectrum relative to the $v=1$ masers at all three epochs. In addition to computing values for individual transitions and epochs, we also combined the three epochs and computed a mean LOS velocity for each transition. We obtained

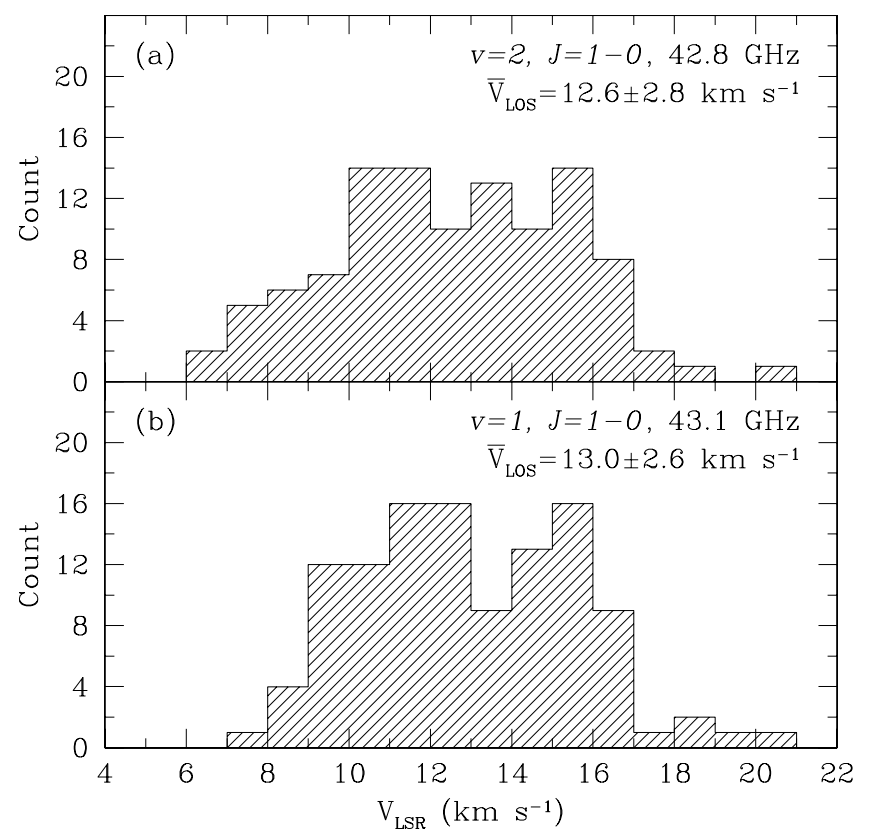

Fig. 9. Histogram of the LOS velocities of our $\mathrm{SiO}$ maser spots combining VLBA epochs A-C. Panel a) shows the histogram for the $v=2$ $42.8 \mathrm{GHz}$ masers and panel b) for the $v=143.1 \mathrm{GHz}$ masers. Also indicated are the mean values $\bar{V}_{v=1}$ and $\bar{V}_{v=2} . \bar{V}_{v=1}$ is slightly redder than $\bar{V}_{v=2}$.

mean values of $V_{\mathrm{LSR}}=12.6 \pm 2.8 \mathrm{~km} \mathrm{~s}^{-1}$ and $13.0 \pm 2.6 \mathrm{~km} \mathrm{~s}^{-1}$ for the $42.8 \mathrm{GHz}$ and $43.1 \mathrm{GHZ}$ masers, respectively. These values are shown in Fig. 9. Again we see that $\bar{V}_{v=1}$ is slightly redder than $\bar{V}_{v=2}$.

To verify the notion that the higher-velocity masers are found closer to the star, we plotted component distance from the center of the distribution (radius) versus its velocity relative to the average LOS velocity for that transition $\left(V_{\mathrm{LOS}}-\bar{V}_{\mathrm{LOS}}\right)$. The results are shown in Figs. 10 and 11 for the $v=1, J=1-043.1 \mathrm{GHz}$ and the $v=2, J=1-042.8 \mathrm{GHz}$ transitions, respectively. In the figures we plotted each epoch with a different symbol. The masers in both transitions appear to have the same distribution with a central peak near $V_{\mathrm{LOS}}-\bar{V}_{\mathrm{LOS}}=0$ and decreasing maser radius as a function of increasing values of $\left|V_{\mathrm{LOS}}-\bar{V}_{\mathrm{LOS}}\right|$.

A simple model that is often used in the case of $\mathrm{OH}$ (e.g. Reid et al. 1977) and $\mathrm{H}_{2} \mathrm{O}$ (e.g. Yates \& Cohen 1994) maser kinematics is that of a uniformly expanding thin shell. In this model the projected distance from center $(a)$ of a maser on the shell is related to its LOS velocity $V-V_{\star}$ by the expression:

$\left(\frac{a}{r_{\mathrm{sh}}}\right)^{2}+\left(\frac{V-V_{\star}}{V_{\mathrm{sh}}}\right)^{2}=1$,

where $r_{\mathrm{sh}}$ and $V_{\mathrm{sh}}$ are the radius and the expansion velocity of the shell, respectively. The LSR velocity of the $\operatorname{star}\left(V_{\star}\right)$ is assumed here to be equal to $\bar{V}_{\text {LOS }}$. Numerical simulations of SiO masers often employ the Sobolev or large velocity gradient (LVG) approximation (Lockett \& Elitzur 1992; Bujarrabal 1994; Doel et al. 1995; Humphreys et al 2002). The LVG approximation assumes a large velocity gradient across the $\mathrm{SiO}$ maser region, thus producing large Doppler shifts that serve to disconnect a region of velocity coherence from the surrounding medium (Bujarrabal 1994). The parameter typically used to characterize the velocity field in the maser region is the logarithmic velocity gradient $\epsilon$ given by

$\epsilon=\frac{\mathrm{d} \ln V}{\mathrm{~d} \ln r}=\frac{r}{V} \frac{\mathrm{d} V}{\mathrm{~d} r}$ 


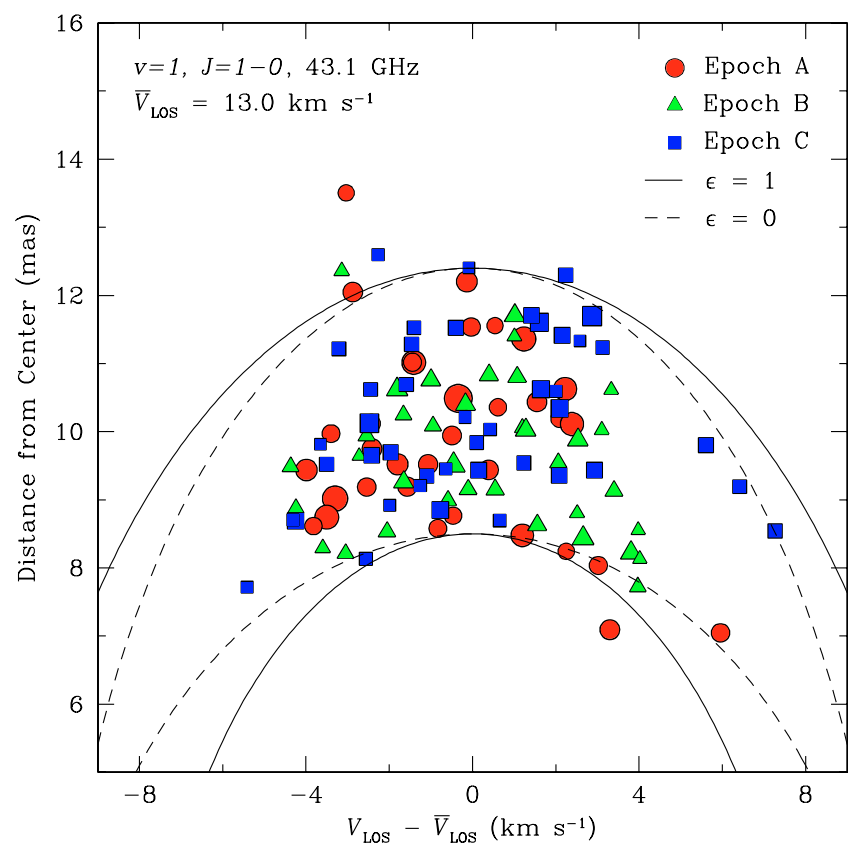

Fig. 10. Distances of the $43.1 \mathrm{GHz}$ maser components from the common center of the distribution (radius) versus their velocity relative to the average LOS velocity for the $43.1 \mathrm{GHz}$ transition $\left(V_{\mathrm{LOS}}-\bar{V}_{\mathrm{LOS}}\right)$. Maser components of the different epochs are denoted by different symbols and colors. The plot indicates that higher-velocity masers are found closer to the star. This trend can be explained by means of expanding maser shells. The curves represent shells at the inner and outer boundaries of the $\mathrm{SiO}$ maser shell using (solid lines) a logarithmic velocity gradient and (dashed lines) constant velocity expansion. See text for details.

A value of $\epsilon=0$ is equivalent to constant velocity expansion, while $\epsilon \geq 1$ corresponds to a velocity field with large radial accelerations. The $\mathrm{SiO}$ maser numerical simulations (e.g. Lockett \& Elitzur 1992; Bujarrabal 1994; Doel et al. 1995) typically use a value of $\epsilon=1$. Chapman \& Cohen (1986) determined values of $\epsilon$ for the various maser species surrounding the supergiant VX Sgr and found $\epsilon \approx 1$ in the $\mathrm{SiO}$ region, $\epsilon \approx 0.5$ in the region of the $\mathrm{H}_{2} \mathrm{O}$ and mainline $(1665 \mathrm{MHz}, 1667 \mathrm{MHz}) \mathrm{OH}$ masers, and $\epsilon \approx 0.2$ in the $1612 \mathrm{MHz} \mathrm{OH}$ maser region.

Plotted along with the data in Figs. 10 and 11 are four curves computed using the above equations. They represent shells at the inner and outer boundaries of the $\mathrm{SiO}$ masers. For the masers in each transition, we used an inner shell radius equivalent to the smallest average distance minus its standard deviation from Table 6. Similarly, for the outer shell radius we used the largest average distance plus its standard deviation from Table 6. For the scenario of a logarithmic velocity gradient, the velocity at the inner $v=2$ boundary was set at $7 \mathrm{~km} \mathrm{~s}^{-1}$ and the velocities of the other shells computed assuming $\epsilon=1$. The velocity of the outermost shell of the $v=1$ transition is $V_{\mathrm{sh}} \approx 10.5 \mathrm{~km} \mathrm{~s}^{-1}$.

For the scenarion of a constant velocity expansion $(\epsilon=0)$, the best by-eye fit results in $V_{\mathrm{sh}}=10 \mathrm{~km} \mathrm{~s}^{-1}$. In this case, the higher expansion velocity at the inner boundary of the $v=2$ transition is required in order to provide a reasonable fit to the outer boundaries of both transitions. For the $\epsilon=1$ case, the inner $v=2$ velocity can be lower with the radial acceleration allowing for a better fit to the outer boundaries. For comparison, the escape velocities computed for the innermost $v=2$ and outermost $v=1$ shells, assuming a mass for S Ori of $1.0 M_{\odot}$, are 22.1 and $18.0 \mathrm{~km} \mathrm{~s}^{-1}$, respectively. Therefore, even at the velocities

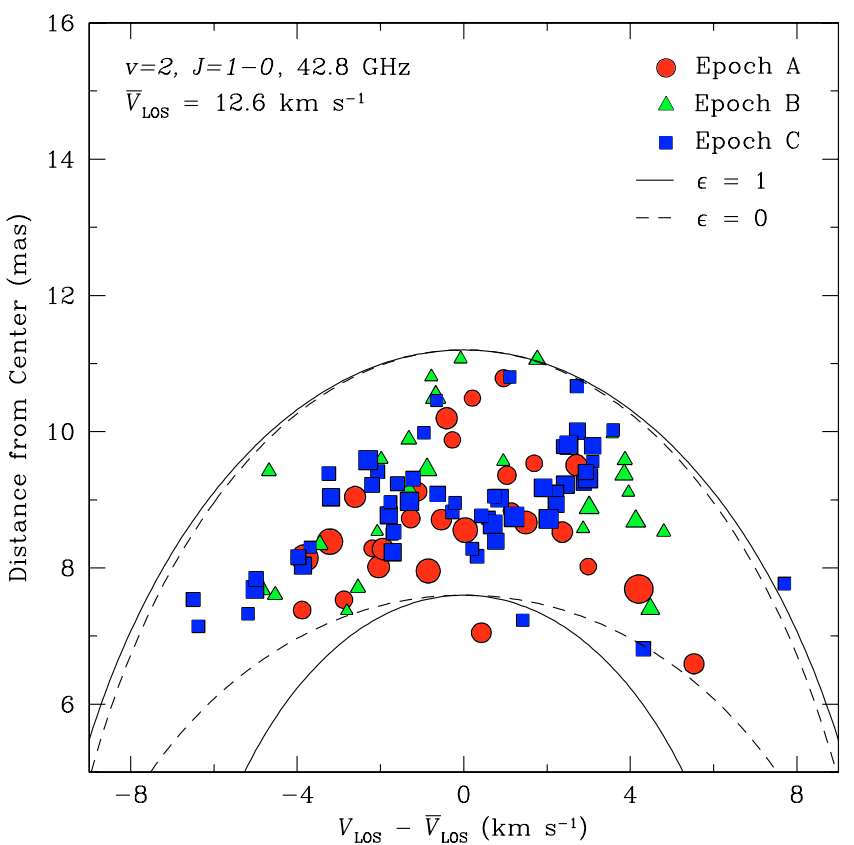

Fig. 11. As Fig. 10, but for the $42.8 \mathrm{GHz}$ transition. The axes' scales are identical for the two figures. Compared to Fig. 10, it is evident that the $42.8 \mathrm{GHz}$ maser spots lie at systematically closer distances to the center of the common maser distribution than the $43.1 \mathrm{GHz}$ maser spots.

bracketed by the curves, the maser gas is still gravitationally bound.

From the $\mathrm{SiO}$ maser data on $\mathrm{S}$ Ori, we cannot definitively rule out constant velocity expansion; however, the $\mathrm{SiO}$ maser region is very dynamic, influenced by the pulsation of the star and the passage of shocks. Theoretical studies all seem to include some form of velocity gradient across the $\mathrm{SiO}$ maser region. In addition, constant velocity expansion would be more conducive to masers along the line of sight to the star because of the increased velocity coherence. $\mathrm{SiO}$ masers are rarely observed in front of the stellar disc, and for S Ori in particular, such masers have not be seen. We therefore consider some form of acceleration in the region the more likely scenario.

The canonical interpretation of the rings formed by the $\mathrm{SiO}$ masers is that the masers are tangentially amplified and primarily confined to a region close to the plane of the sky. Under this interpretation, the ring size and thickness are direct indicators of the equivalent 3-D shell size and thickness. The radially expanding spherical shell described above will naturally result in a projection effect where masers out of the plane of the sky have smaller angular distances from center due to the projection. In the case of S Ori, this projection is rather small. The equation for the angle $\theta$ measured between the LOS and a point on the shell locating the maser can be written in terms of the velocities as

$\sin \theta=\left[1-\left(\frac{V}{V_{\mathrm{sh}}}\right)^{2}\right]^{1 / 2}$

In the most extreme case, all of the thickness observed in the ring is due to projection of the masers on the outermost thin shell with a velocity of $V_{\mathrm{sh}} \approx 10.5 \mathrm{~km} \mathrm{~s}^{-1}(\epsilon=1)$. Figures 9-11 show that the majority of the masers are within $\sim 4 \mathrm{~km} \mathrm{~s}^{-1}$ of LOS center velocity. For these extreme $4 \mathrm{~km} \mathrm{~s}^{-1}$ masers, $\sin \theta \approx 0.92$ and $\theta \approx 67^{\circ}$. This results in a difference of $\sim 8 \%$ between the projected distance of the maser and its true radial distance. For masers less than $4 \mathrm{~km} \mathrm{~s}^{-1}$ the difference is smaller. Assuming the masers are distributed uniformly, the differences between the 
Table 7. Overview of the photometric and spatial parameters of S Ori as a function of stellar phase, derived from both our VLTI and VLBA observations.

\begin{tabular}{lrrrr}
\hline \hline & $\mathrm{A}$ & $\mathrm{B}$ & $\mathrm{C}$ & $\mathrm{D}$ \\
\hline Phase $\Phi_{\text {vis }}$ & 0.44 & 0.55 & 1.15 & 1.27 \\
$V[\mathrm{mag}]$ & $12.8 \pm 0.3$ & $14.0 \pm 0.3$ & $9.4 \pm 0.3$ & $10.5 \pm 0.4$ \\
$f_{N}[\mathrm{Jy}]$ & $187 \pm 12$ & $152 \pm 17$ & $198 \pm 18$ & $203 \pm 13$ \\
$f_{43.1}[\mathrm{Jy}]$ & $119 \pm 18$ & $203 \pm 30$ & $198 \pm 30$ & \\
$f_{42.8}[\mathrm{Jy}]$ & $148 \pm 22$ & $46 \pm 7$ & $383 \pm 57$ & \\
$\Theta_{\text {Phot }}[\mathrm{mas}]$ & $9.0 \pm 0.3$ & $7.9 \pm 0.1$ & $9.7 \pm 0.1$ & $9.5 \pm 0.4$ \\
$\Theta_{\text {in }}[\mathrm{mas}]$ & $16.2 \pm 1.9$ & $15.9 \pm 1.6$ & $21.2 \pm 1.9$ & $22.8 \pm 2.1$ \\
$\Theta_{43.1}[\mathrm{mas}]$ & $19.6 \pm 2.8$ & $19.0 \pm 2.6$ & $20.4 \pm 2.4$ & \\
$\Theta_{42.8}[\mathrm{mas}]$ & $19.0 \pm 2.0$ & $17.8 \pm 3.4$ & $17.8 \pm 1.8$ & \\
$\Theta_{\text {in }} / \Theta_{\text {Phot }}$ & $1.8 \pm 0.2$ & $2.0 \pm 0.2$ & $2.2 \pm 0.2$ & $2.4 \pm 0.2$ \\
$\Theta_{43.1} / \Theta_{\text {Phot }}$ & $2.2 \pm 0.3$ & $2.4 \pm 0.3$ & $2.1 \pm 0.3$ & \\
$\Theta_{42.8} / \Theta_{\text {Phot }}$ & $2.1 \pm 0.2$ & $2.3 \pm 0.4$ & $1.9 \pm 0.2$ & \\
$\Theta_{43.1} / \Theta_{\text {in }}$ & $1.2 \pm 0.2$ & $1.2 \pm 0.2$ & $1.0 \pm 0.1$ & \\
$\Theta_{42.8} / \Theta_{\text {in }}$ & $1.2 \pm 0.2$ & $1.1 \pm 0.2$ & $0.8 \pm 0.1$ & \\
$R_{\text {Phot }}\left[R_{\odot}\right]$ & $469 \pm 127$ & $411 \pm 111$ & $498 \pm 134$ & $491 \pm 133$ \\
\hline
\end{tabular}

projected ring sizes and thicknesses and their 3-D equivalents is $\sim 4 \%$. Thus, the given errors of the projected ring sizes determined from the maser distributions include the uncertainty of the projection effect.

\section{VLTI/MIDI and VLBA results as a function of stellar phase}

Table 7 provides an overview on the photometric and spatial parameters of S Ori as a function of observational epoch/stellar phase, as derived from both our VLTI/MIDI and VLBA observations in Sects. 3 and 4. The listed photometric parameters include the $V$ magnitude based on the lightcurve in Fig. 1, the $N$-band flux based on our photometric MIDI data (Sect. 3.1), and the total flux values of the two maser transitions (Sect. 4.1). The listed spatial information includes the photospheric angular diameter $\Theta_{\text {Phot }}$ (Sect. 3.4), the inner dust shell diameter $\Theta_{\text {in }}$ (Sect. 3.4), and the maser ring angular diameters $\Theta_{43.1}$ and $\Theta_{42.8}$ (Sect. 4.2.1). To compare the continuum photospheric diameter, the inner dust shell diameter, and the maser shell diameters, the ratios $\Theta_{\text {in }} / \Theta_{\text {Phot }}, \Theta_{43.1 / 42.8} / \Theta_{\text {Phot }}$, and $\Theta_{43.1 / 42.8} / \Theta_{\text {in }}$ are listed as well. As an absolute scale, the stellar photospheric radius $R_{\text {Phot }}$ at each phase is derived from $\Theta_{\text {Phot }}$ and the adopted distance to S Ori (Sect. 2). Here, the variability phases are the mean values of the respective phases of the MIDI and VLBA observations.

The continuum photospheric radii $R_{\text {Phot }}$, the inner dust shell radii $R_{\text {in }}$ (corresponding to $\Theta_{\text {in }}$ ) and the maser shell radii $R_{43.1}$ and $R_{42.8}$ (corresponding to $\Theta_{43.1 / 42.8}$ ) are indicated in panels (d) of Figs. 2-5, which show the synthetic mid-infrared CLVs. This allows a comparison of these radii to each other and to the profile of the extended molecular atmosphere. Figure 7 provides as well a graphical comparison of the photospheric disc, the molecular layers, the dust shell, and the maser spots for each epoch.

Variability of visual, mid-infrared, and maser flux densities The visual, mid-infrared, and maser fluxes all show clear variability as a function of observational epoch. The stellar phases given throughout this paper are visual phases as introduced in Sect. 2. The $N$-band flux $f_{N}$ shows clear variability with an amplitude of about $50 \mathrm{Jy}(\sim 30 \%) . f_{N}$ exhibits a minimum at our epoch B at visual phase 0.55 , i.e. shortly beyond the visual minimum, rises toward stellar maximum, and continues to increase between epochs $\mathrm{C}$ and $\mathrm{D}$ at visual phases 1.15 and 1.27, probably indicating a phase lag and different shape of the $N$ band variability curve compared to the visual lightcurve. The total maser fluxes are significantly lower near stellar minimum (epochs A and B) compared to stellar maximum (epoch C), with the exception of the strong $f_{43.1}$ value at epoch $\mathrm{B}$. The latter can most likely be explained by the larger number of maser spots forming a nearly complete ring-like structure for this transition and epoch, while the other transitions and epochs show only partial maser rings.

Photospheric and dust shell radii as a function of visual phase The photospheric angular diameter $\Theta_{\text {Phot }}$ derived from our MIDI observations and modeling shows a clearly phasedependent size with an amplitude of about 1.8 mas $(\sim 20 \%)$ that is well-correlated in phase with the visual lightcurve. It shows a minimum at epoch $\mathrm{B}$, which is nearest to the visual minimum, and a maximum at epoch $\mathrm{C}$, which is nearest to the visual maximum. Also the inner dust-shell radius shows a variation (amplitude $\sim 7$ mas $\sim 30 \%$ ), which is more closely correlated in phase and amplitude to $f_{N}$ than to $V$. The inner dust shell radius is smallest at our epoch $\mathrm{B}$ near visual minimum and largest at epoch D beyond visual maximum (phase 1.27). Compared to $\Theta_{\mathrm{Phot}}$, the inner dust shell boundary is located significantly closer to the stellar surface at our epochs A and B near visual minimum $\left(1.8-2.0 \times \Theta_{\text {Phot }}\right)$ than at the post-maximum visual phases C and D (2.2-2.4 $\left.\times \Theta_{\text {Phot }}\right)$. Simultaneously, as discussed in Sect. 3.4, the density gradient $p$ is significantly steeper and the optical depth significantly higher near visual minimum than at the post-maximum visual phases. This can be understood by a wind model with increased mass loss near minimum phase occurring close to the stellar surface and an expanded dust shell after maximum (cf. Sect. 3.4).

Maser ring radii as a function of visual phase The mean $\mathrm{SiO}$ maser ring radii obtained at our three VLBA epochs show a small variability with amplitude of $\leq 7 \%$, which is only a fraction of the mean width of the maser shells of $\sim 13 \%$ and clearly below the variability amplitude of the photospheric and dust shell radii at the same epochs. However, we cannot rule out that the maser ring radii show a greater variability in between our epochs of observation. Observations by Cotton et al. (2006) consistently find variations in maser ring diameters of 3-14\% for different Mira stars, but no clear correlation with visual variability phase. Theoretical estimates by Humphreys et al. (2002) predict a variability of the $43.1 \mathrm{GHz}$ maser shell radius with an amplitude of $\sim 20 \%$.

Maser ring radii compared to the photospheric radius The true location of the star relative to the maser spots is unknown. Based on the assumption that the center of the maser spot distribution coincides with the center of the star, a comparison of the maser ring radii with the continuum photospheric radii gives an estimate of the distance of the maser spots from the stellar photosphere. The ratios between the maser ring radii and the photospheric radii in Table 7 range between $2.1 \pm 0.3$ and $2.4 \pm 0.3$. These values are consistent with the values reported in BW05 (1.9 and 2.0), with those by Cotton et al. (2004), which range for different Mira stars - not always compared at the same phase between 1.8 and 2.9, and the value by Fedele et al. (2005) for 
$\mathrm{R}$ Leo of 1.8. Our observations indicate a variability in the ratio of the mean maser shell radius to the photospheric radius with an amplitude of $\sim 10 \%$ for the $43.1 \mathrm{GHz}$ transition, and $\sim 20 \%$ for the $42.8 \mathrm{GHz}$ transition, with uncertainties on the same order. This observed variability is dominated by the variability of the stellar continuum photospheric diameter.

Maser ring radii compared to the molecular atmosphere Panels (d) of Figs. 2-5 as well as Fig. 7 show that the mean maser ring radii at all our epochs mark the region just beyond the steepest decrease in the mid-infrared model intensity, i.e. shortly outward of the densest region of the molecular atmosphere.

Maser ring radii compared to the inner dust shell boundary Table 7, as well as panels (d) of Figs. 2-5, indicate that the inner boundary of the $\mathrm{Al}_{2} \mathrm{O}_{3}$ dust grains near visual minimum (epochs $\mathrm{A}$ and $\mathrm{B}$ ) appears to be located between the steepest decrease in the mid-infrared model intensity and the mean location of the $\mathrm{SiO}$ maser spots. This means that the lowdensity atmospheric molecular layers, the $\mathrm{SiO}$ maser spots, and the $\mathrm{Al}_{2} \mathrm{O}_{3}$ dust grains are co-located near visual minimum. At our post-maximum epochs $\mathrm{C}$ and $\mathrm{D}$, the inner boundary of the $\mathrm{Al}_{2} \mathrm{O}_{3}$ dust shell appears significantly expanded, while the maser shells remain at about the same location. As a result, the inner boundary of the $\mathrm{Al}_{2} \mathrm{O}_{3}$ dust grains appears to have expanded outward of the mean $\mathrm{SiO}$ maser rings at the post-maximum phase.

\section{Summary, conclusions, and discussion}

We have obtained the first time series of observations of a Mira variable star that include concurrent infrared and radio interferometry. For the present paper, we obtained mid-infrared interferometry of S Ori with VLTI/MIDI at four epochs in December 2004, February/March 2005, November 2005, and December 2005. We observed $v=1, J=1-0(43.1 \mathrm{GHz})$ and $v=2, J=1-0(42.8 \mathrm{GHz}) \mathrm{SiO}$ maser emission toward S Ori with the VLBA in January, February, and November 2005, concurrent to within $5 \%$ of the variability period to the first three VLTI/MIDI epochs. The first two common VLTI/VLBA epochs are located near visual minimum (phases 0.44 and 0.55 ) and the third after next visual maximum (phase 1.15). The fourth MIDI epoch occurred at a later post-maximum phase of 1.27.

The mid-infrared MIDI data are sensitive to the structure of the atmosphere consisting of the continuum photosphere and overlying molecular layers, as well as to the properties of the dust shell. The MIDI visibilities and flux densities are modeled using the recent $\mathrm{M}$ model atmosphere series by Ireland et al. (2004b), which is a dust-free self-excited dynamic model atmosphere series, to which we add an ad-hoc radiative transfer calculation of the dust shell. The constrained model parameters include the continuum photospheric angular diameter, as well as characteristics of the dust shell. The MIDI visibility and total flux data at all epochs can be described well by the chosen approach of combining the dust-free $M$ model atmosphere series with an ad-hoc radiative transfer model of the dust shell. Different model parameters can be constrained by different subsets of our MIDI data.

The resulting continuum photospheric angular diameters at visual variability phases $0.42,0.55,1.16$, and 1.27 are $9.0 \pm 0.3$ mas, $7.9 \pm 0.1$ mas, $9.7 \pm 0.1$ mas, and $9.5 \pm 0.4$ mas, respectively. The photospheric radius thus shows a significantly phase-dependent size with amplitude of $\sim 20 \%$ that is well correlated in phase with the visual lightcurve.
The dust shell can be modeled well with $\mathrm{Al}_{2} \mathrm{O}_{3}$ grains alone, and our data show no indication of silicates, consistent with the modeling of the IRAS LRS spectra by Lorenz-Martins \& Pompeia (2000). The inner dust shell angular diameters are $16.2 \pm 1.9$ mas, $15.9 \pm 1.6$ mas, $21.2 \pm 1.9$ mas, and $22.8 \pm 2.1$ mas, respectively, for the visual phases given above, corresponding to $1.8,2.0,2.2$, and 2.4 times the continuum photospheric diameters. Thus, dust starts to form very close to the stellar surface, corresponding to the region of the low-intensity wings of the atmospheric molecular layers of our dust-free atmosphere model. In particular, the inner dust shell radius is smallest at the near-minimum phases, located directly outside of the steep decrease in the dust-free, mid-infrared model intensity. The inner dust-shell boundary expands to larger distances from the stellar surface at our post-maximum phases. The overall $\mathrm{Al}_{2} \mathrm{O}_{3}$ optical depth, as well as the density gradient, is largest at our nearminimum phases and significantly lower at the post-maximum stellar phases. These dust shell characteristics can be explained by a radiatively driven wind with increased mass-loss near visual stellar minimum occurring close to the stellar surface, and an expanded dust shell at the post-maximum phases.

The $43.1 \mathrm{GHz}$ and $42.8 \mathrm{GHz}$ maser spots show the typical structure of partial to full rings with a clumpy distribution. There is no sign of a globally asymmetric gas distribution. The $43.1 \mathrm{GHz}$ mean maser ring angular diameters at visual phases $0.46,0.56$, and 1.14 are $19.6 \pm 2.8$ mas, $19.0 \pm 2.6$ mas, and $20.4 \pm 2.4$ mas, respectively. The values for the $42.8 \mathrm{GHz}$ maser are systematically smaller by $3-19 \%$ and amount to $19.0 \pm 2.0$ mas, $17.8 \pm 3.4$ mas, and $17.8 \pm 1.8$ mas. The indication of systematically smaller mean maser ring diameters of the $42.8 \mathrm{GHz}$ transition compared to the $43.1 \mathrm{GHz}$ transition is consistent with earlier results in the literature on other Mira stars. The velocity structure of both maser transitions indicate an expansion of the maser shell. This expansion is most likely an accelerated expansion, but we cannot completely rule out a uniform expansion. The expansion velocity for both transitions ranges between about $7 \mathrm{~km} \mathrm{~s}^{-1}$ and about $10 \mathrm{~km} \mathrm{~s}^{-1}$ between the inner and outer shell radii based on a scenario of an accelerated expansion or amounts to about $10 \mathrm{~km} \mathrm{~s}^{-1}$ based on a scenario with uniform expansion.

Compared to the continuum photospheric radius $R_{\text {Phot }}$, the $43.1 \mathrm{GHz} / 42.8 \mathrm{GHz} \mathrm{SiO}$ maser ring radii are located at $2.2 \pm 0.3 / 2.1 \pm 0.2$ (phase 0.44), $2.4 \pm 0.3 / 2.3 \pm 0.4(0.55)$, and $2.1 \pm 0.3 / 1.9 \pm 0.2(1.15)$ times $R_{\text {Phot }}$. These values are consistent with theoretical estimates and earlier observations available in the literature. Compared to the molecular atmosphere, the maser shells mark the region of the molecular atmosphere just beyond the steepest decrease in the mid-infrared model intensity. Compared to the dust shell, the maser spots are co-located with the inner dust shell near stellar minimum. At our post-maximum phases, the maser spots remain at about the same location, while the dust shell has expanded outward.

Altogether, our observations indicate a pulsation of the stellar photosphere approximately in phase with the visual lightcurve. Our measurements suggest an increased mass-loss rate near stellar minimum and formation of $\mathrm{Al}_{2} \mathrm{O}_{3}$ dust grains occurring close to the stellar surface at about 1.8-2.0 photospheric radii directly outward of the dense molecular atmosphere, co-located with the extended low-intensity wings of the molecular atmosphere and with the $\mathrm{SiO}$ maser spots. At the post-maximum phases, the dust shell has expanded to about 2.2-2.4 photospheric radii, while the mean radius of the maser spots remains at $\sim 2.0$ photospheric radii. 


\section{S Ori, near visual minimum}

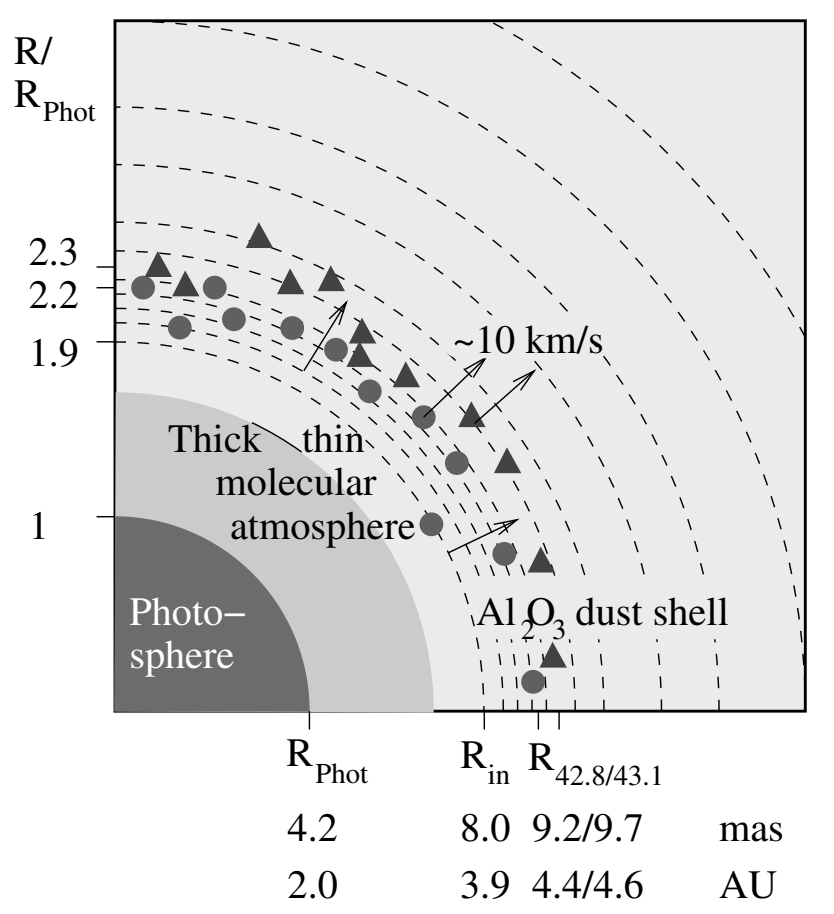

S Ori, post visual maximum

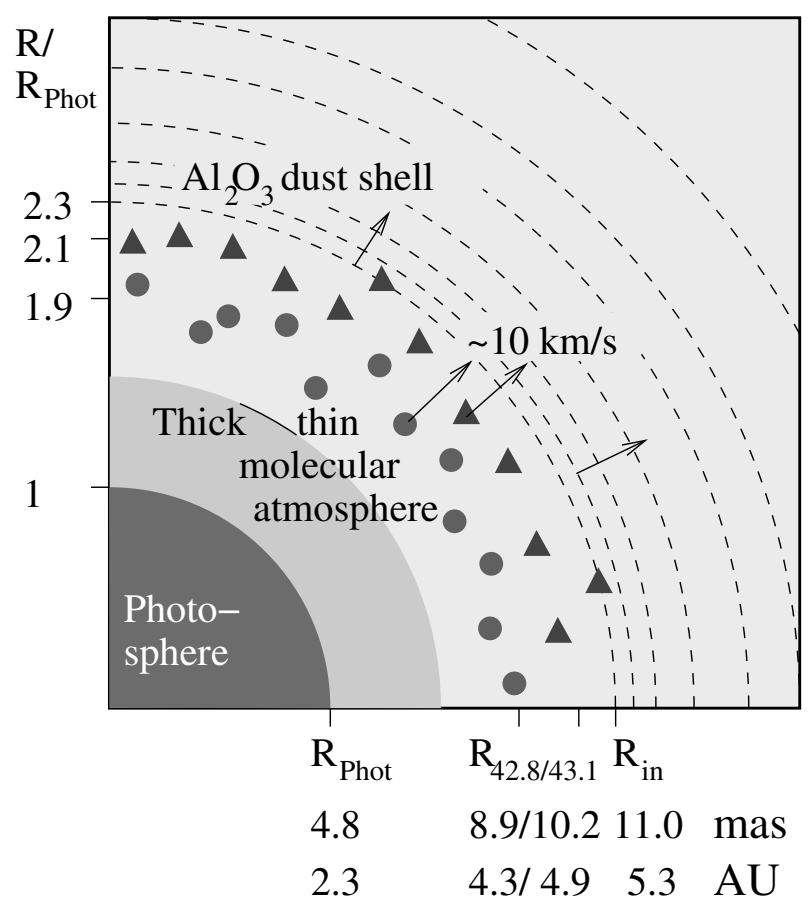

Fig. 12. Sketch of the radial structure of S Ori's CSE at (left) near-minimum and (right) post-maximum visual phase as derived in this work. Shown are the locations of the continuum photosphere (dark gray), the at $N$-band optically thick molecular atmosphere (medium dark gray), the at $N$-band optically thin molecular atmosphere (light gray), the $\mathrm{Al}_{2} \mathrm{O}_{3}$ dust shell (dashed arcs), and the $42.8 \mathrm{GHz}$ and $43.1 \mathrm{GHz}$ maser spots (circles/triangles). The numbers below and beside the panels are the mean values of (left) epochs A \& B and (right) epochs C \& D from Table 7 .

Figure 12 shows a sketch of S Ori and its CSE near visual minimum and post-maximum illustrating the findings of the present work. Our results on S Ori are largely consistent with the canonical scheme of the CSE of AGB stars discussed for instance in Reid \& Menten (1997). However, our results indicate an inner dust shell boundary that is located closer to the optical photosphere compared to the Reid \& Menten scheme at about two photospheric radii, and $\mathrm{SiO}$ maser shells that are colocated with the low-intensity molecular atmosphere and with $\mathrm{Al}_{2} \mathrm{O}_{3}$ dust grains near visual minimum. In the case of $\mathrm{S}$ Ori, the dust shell consists only of $\mathrm{Al}_{2} \mathrm{O}_{3}$ grains. For Mira stars that also show a dust shell of silicates, the inner boundary of silicate grains is still expected at greater distances of $\gtrsim 4$ photospheric radii (Lorenz-Martins \& Pompeia 2000). In addition to a static picture of a Mira star's CSE, our observations suggest the formation of a dust shell close to the surface near minimum phase and an expansion by $\sim 20 \%$ of the inner dust-shell boundary toward post-maximum phase.

Our modeling approach of adding the CLVs of a dust-free $\mathrm{M}$ model to that of a radiative transfer model of the pure dust shell includes two uncertainties. Firstly, the M model series is designed for the prototype Miras $o$ Cet and R Leo and used here for the longer-period Mira S Ori. Secondly, we omit a possible interaction of gas and dust outward of the inner dust shell boundary. Both effects might add some additional uncertainty to the absolute values of the obtained photospheric diameter values and dust shell characteristics. However, our data can be described well by our modeling approach and these additional uncertainties are not expected to be significantly larger than the diameter errors given in Table 7 and the step sizes of the dust shell parameters in Table 4. Furthermore, the relative accuracy of the obtained parameters among the four epochs of observations are clearly less affected by possible systematic uncertainties. Currently, the chosen approach appears to be the best available one. Similar studies of S Ori and other long-period Mira stars in the future would benefit from new dynamic model atmosphere series tailored to Mira stars with a longer period and a higher mass compared to $o$ Cet and R Leo, as well as from self-consistent calculations of dynamic atmospheres and dust formation.

Our mid-infrared interferometric data, in particular at the lower edge of MIDI's wavelength range, together with the use of the M model series provide constraints on the continuum photospheric angular diameter of S Ori. The resulting values are in good agreement with several earlier diameter measurements obtained in the near-infrared $K$-band by different instruments and at different stellar phases, which increases the confidence in our modeling approach. Additional confidence in the continuum photospheric diameters and the validity of the dynamic model atmospheres can in future be reached by time series of high spectral resolution $(R=1000-10000)$ near-infrared interferometry with the VLTI/AMBER (Petrov et al. 2003) instrument concurrent to VLTI/MIDI observations.

Our results obtained at 4 epochs within one pulsation cycle suggest the formation of a dust layer consisting of $\mathrm{Al}_{2} \mathrm{O}_{3}$ grains close to the stellar surface near stellar minimum and an expanded dust shell after stellar maximum. Our understanding of the connection between the dust formation frequency and the stellar pulsation will be further improved by using more epochs within one pulsation cycle. Dust-forming episodes may also have their own timescale that may not be directly related to the visual variability (e.g., Höfner \& Dorfi 1997; Nowotny et al. 2005), a study of which requires observations extended over more than one pulsation cycle. Since the data of S Ori do not show any sign of silicate grains, similar observations of targets that belong to the 
intermediate class group of Lorenz-Martins \& Pompeia (exhibiting $\mathrm{Al}_{2} \mathrm{O}_{3}$ and silicate grains) would be required to study the detailed relationship among the $\mathrm{Al}_{2} \mathrm{O}_{3}$ shell, the silicate shell, and the photospheric pulsation.

Both our MIDI data and the images of the $\mathrm{SiO}$ maser shell do not show any signature of a globally asymmetric distribution of dust or gas in the case of S Ori within our uncertainties. In general, asymmetric distributions of gas and dust are to be expected for some Mira stars (e.g., Weigelt et al. 1996; Boboltz \& Diamond 2005; Tatebe et al. 2006). A detection of asymmetric structures can be performed at the VLTI by sequentially using baselines of similar length but different orientation close in time/stellar phase to MIDI and by using the closure-phase instrument AMBER with baseline triangles comprised of baselines with different orientation.

The relative positions of star and $\mathrm{SiO}$ maser shells in our current study is unknown. Our comparison of the extension of the $\mathrm{SiO}$ maser shells to the continuum photospheric diameter is based on the assumption that the center of the maser spot distribution coincides with the center of the star. This assumption is the most likely scenario for stars that do not show any sign of asymmetric dust and gas distributions, such as S Ori. In principle, the relative position of the maser shells could be related relative to the radio photosphere, which was detected by Reid \& Menten (1997) for a sample of 6 Mira and semi-regular variables. Additional measurements of $\mathrm{H}_{2} \mathrm{O}$ and $\mathrm{OH}$ masers relative to the $\mathrm{SiO}$ masers could give additional information on the structure and kinematics of the more extended CSE.

Acknowledgements. We thank Walter Jaffe, Rainer Köhler, Christian Hummel, and others involved for making publicly available the MIDI data reduction software packages and tools EWS, MIA, and MyMidiGui, as well as for helpful discussions on MIDI data reduction. We are grateful for support by the ESO DGDF. The work of MS was supported by a grant of the Deutsche Forschungsgemeinschaft on "Time Dependence of Mira Atmospheres". We acknowledge with thanks the variable-star observations from the AAVSO International Database contributed by observers worldwide and used in this research. This research has made use of the AFOEV and SIMBAD databases, operated at the CDS, France. This research has made use of NASA's Astrophysics Data System.

\section{References}

Begemann, B., Dorschner, J., Henning, T., et al. 1997, ApJ, 476, 199

Beichman, C. A., Neugebauer, G., Habing, H. J., Clegg, P. E., \& Chester, T. J. 1988, Infrared astronomical satellite (IRAS) catalogs and atlases Benson, P. J., Little-Marenin, I. R., Woods, T. C., et al. 1990, ApJS, 74, 911 Boboltz, D. A., \& Marvel, K. B. 2000, ApJ, 545, L149

Boboltz, D. A., \& Diamond, P. J. 2005, ApJ, 625, 978

Boboltz, D. A., \& Wittkowski, M. 2005, ApJ, 618, 953 (BW05)

Boboltz, D. A., Diamond, P. J., \& Kemball, A. J. 1997, ApJ, 487, L147

Bujarrabal, V. 1994, A\&A, 285, 953

Chapman, J. M., \& Cohen, R. J. 1986, MNRAS, 220, 513

Cho, S.-H., Kaifu, N., \& Ukita, N. 1996, AJ, 111, 1987

Cohen, M., Walker, R. G., Carter, B., et al. 1999, AJ, 117, 1864

Cotton, W. D., Mennesson, B., Diamond, P. J., et al. 2004, A\&A, 414, 275

Cotton, W. D., Vlemmings, W., Mennesson, B., et al. 2006, A\&A, 456, 339

Danchi, W. C., Bester, M., Degiacomi, C. G., Greenhill, L. J., \& Townes, C. H. 1994, AJ, 107, 1469

Desmurs, J. F., Bujarrabal, V., Colomer, F., \& Alcolea, J. 2006, A\&A, 360, 189

Diamond, P. J., \& Kemball, A. J. 2003, ApJ, 599, 1372

Diamond, P. J., Kemball, A. J., Junor, W., et al. 1994, ApJ, 430, L61

Doel, R. C., Gray, M. D., Humphreys, E. M. L., Braithwaite, M. F., \& Field, D. 1995, A\&A, 302, 797

Feast, M. W., Glass, I. S., Whitelock, P. A., \& Catchpole, R. M. 1989, MNRAS, 241,375

Fedele, D., Wittkowski, M., Paresce, F., et al. 2005, A\&A, 431, 1019

Glindemann, A., Algomedo, J., Amestica, R., et al. 2003, Proc. SPIE, 4838, 89
Gray, M. D., \& Humphreys, E. M. L. 2000, New Astron., 5, 155

Haniff, C. A., Scholz, M., \& Tuthill, P. G. 1995, MNRAS, 276, 640

Henden, A. A. 2006, Observations from the AAVSO International Database, private communication

Höfner, S., \& Dorfi, E. A. 1997, A\&A, 319, 648

Hofmann, K.-H., Scholz, M., \& Wood, P. R. 1998, A\&A, 339, 846

Hofmann, K.-H., Balega, Y., Scholz, M., \& Weigelt, G. 2000, A\&A, 353, 1016 Hofmann, K.-H., Balega, Y., Scholz, M., \& Weigelt, G. 2001, A\&A, 376, 518

Hofmann, K.-H., Beckmann, U., Blöcker, T., et al. 2002, New Astron., 7, 9

Hollis, J. M., Boboltz, D. A., Pedelty, J. A., White, S. M., \& Forster, J. R. 2001, ApJ, 559, L37

Humphreys, E. M. L., Gray, M. D., Yates, J. A., et al. 1996, MNRAS, 282, 1359

Humphreys, E. M. L., Gray, M. D., Yates, J. A., et al. 2002, A\&A, 386, 256

Ireland, M. J., \& Scholz, M. 2006, MNRAS, 367, 1585

Ireland, M. J., Scholz, M., \& Wood, P. R. 2004a, MNRAS, 352, 318

Ireland, M. J., Scholz, M., Tuthill, P. G., \& Wood, P. R. 2004b, MNRAS, 355, 444

Ireland, M. J., Tuthill, P. G., Davis, J., \& Tango, W. 2005, MNRAS, 361, 337

Ivezić, Ž., \& Elitzur, M. 1997, MNRAS, 287, 799

Ivezić, Ž., Nenkova, M., \& Elitzur, M 1999, User Manual for DUSTY, University of Kentucky Internal Report, accessible at http://www.pa.uky.edu/ moshe/dusty

Jacob, A. P., \& Scholz, M. 2002, MNRAS, 336, 1377

Jeong, K. S., Winters, J. M., Le Bertre, T., \& Sedlmayr, E. 2003, A\&A, 407, 191 Jewell, P. R., Snyder, L. E., Walmsley, C. M., Wilson, T. L., \& Gensheimer, P. D. 1991, A\&A, 242, 211

Jura, M., \& Kleinmann, S. G. 1990, ApJS, 73, 769

Kemball, A. J., \& Diamond, P. J. 1997, ApJ, 481, L111

Koike, C., Kaito, C., Yamamoto, T., et al. 1995, Icarus, 114, 203

Leinert, Ch., Graser, U., Richichi, A., et al. 2003, The Messenger, 112, 13

Leinert, Ch., van Boekel, R., Water, L. B. F. M., et al. 2004, A\&A, 423, 537

Lockett, P., \& Elitzur, M. 1992, ApJ, 399, 704

Lorenz-Martins, S., \& Pompeia, L. 2000, MNRAS, 315, 856

Mennesson, B., Perrin, G., Chagnon, G., et al. 2002, ApJ, 579, 446

Millan-Gabet, R., Pedretti, E., Monnier, J. D., et al. 2005, ApJ, 620, 961

Nowotny, W., Aringer, B., Höfner, S., Gautschy-Loidl, R., \& Windsteig, W. 2005, A\&A, 437, 273

Ohnaka, K., Bergeat, J., Driebe, T., et al. 2005, A\&A, 429, 1057

Ohnaka, K., Driebe, T., Hofmann, K.-H., et al. 2006a, A\&A, 45, 1015

Ohnaka, K., Scholz, M., \& Wood, P. R 2006b, A\&A, 446, 1119

Ohnaka, K., Driebe, T., Weigelt, G., \& Wittkowski, M. 2007, A\&A, 466, 1099

Ossenkopf, V., Henning, Th., \& Mathis, J. S. 1992, A\&A, 261, 567

Perrin, G., Ridgway, S. T., Mennesson, B., et al. 2004, A\&A, 426, 279

Petrov, R., Malbet, F., Weigelt, G., et al. 2003, Proc. SPIE, 4838, 924

Quirrenbach, A., Mozurkewich, D., Armstrong, J. T., et al. 1992, A\&A, 259, L19

Reid, M. J., \& Menten, K. M. 1997, ApJ, 476, 327

Reid, M. J., Muhleman, D. O., Moran, J. M., Johnston, K. J., \& Schwartz, P. R. 1977, ApJ, 214, 60

Samus, N. N., Durlevich, O. V., et al. 2004, Combined General Catalog of Variable Stars (GCVS4.2, 2004 Ed.) VizieR Online Data Catalog, 2250, 0

Scholz, M. 2001, MNRAS, 321, 347

Scholz, M. 2003, Proc. SPIE, 4838, 163

Sloan, G. C., \& Price, S. D. 1998, ApJS, 119, 141

Tatebe, K., Chandler, A. A., Hale, D. D. S., \& Townes, C. H. 2006, ApJ, 652, 666

Tej. A., Lançon, A., \& Scholz, M. 2003a, A\&A, 401, 347

Tej, A., Lançon, A., Scholz, M., \& Wood, P. R. 2003b, A\&A, 412, 481

Templeton, M. R., Mattei, J. A., \& Willson, L. A. 2005, AJ, 130, 788

Thompson, R. R., Creech-Eakman, M. J., \& van Belle, G. T. 2002, ApJ, 577, 447

van Belle, G. T., Dyck, H. M., Benson, J. A., \& Lacasse, M. G. 1996, AJ, 112, 2147

van Belle, G. T., Thompson, R. R., \& Creech-Eakman, M. J 2002, AJ, 124, 1706

Weigelt, G., Balega, Y., Hofmann, K.-H., \& Scholz, M. 1996, A\&A, 316, L21

Weiner, J., Tatebe, K., Hale, D. D. S. et al. 2006, ApJ, 636, 1067

Wilson, R. E. 1953, General catalog of stellar radial velocities, Carnegie Institute Washington D.C

Winters, J. M., Le Bertre, T., Jeong, K. S., Nyman, L.-A., \& Epchtein, N. 2003, A\&A, 409, 715

Wittkowski, M., Aufdenberg, J. P., Driebe, T., et al. 2006, A\&A, 460, 855

Woitke, P. 2006, A\&A, 460, L9

Woodruff, H. C., Eberhardt, M., Driebe, T., et al. 2004, A\&A, 421, 703

Wyatt, S. P., \& Cahn, J. H. 1983, ApJ, 275, 225

Yates, J. A., \& Cohen, R. J. 1994, MNRAS, 270, 958

Yi, J., Booth, R. S., Conway, J. E., \& Diamond, P. J. 2005, A\&A, 432, 531

Young, K. 1995, ApJ, 445, 872 